\title{
Distance-based intuitionistic multiplicative multiple criteria decision-making methods for healthcare management in West China Hospital
}

DOI:

10.1111/exsy. 12479

\section{Document Version}

Accepted author manuscript

Link to publication record in Manchester Research Explorer

Citation for published version (APA):

Liao, H., Zhang, C., Luo, L., Xu, Z., Yang, J. B., \& Xu, D. L. (2020). Distance-based intuitionistic multiplicative multiple criteria decision-making methods for healthcare management in West China Hospital. Expert Systems, [e12479]. https://doi.org/10.1111/exsy.12479

\section{Published in:}

Expert Systems

\section{Citing this paper}

Please note that where the full-text provided on Manchester Research Explorer is the Author Accepted Manuscript or Proof version this may differ from the final Published version. If citing, it is advised that you check and use the publisher's definitive version.

\section{General rights}

Copyright and moral rights for the publications made accessible in the Research Explorer are retained by the authors and/or other copyright owners and it is a condition of accessing publications that users recognise and abide by the legal requirements associated with these rights.

\section{Takedown policy}

If you believe that this document breaches copyright please refer to the University of Manchester's Takedown Procedures [http://man.ac.uk/04Y6Bo] or contact uml.scholarlycommunications@manchester.ac.uk providing relevant details, so we can investigate your claim.

\section{OPEN ACCESS}




\title{
Distance-based intuitionistic multiplicative multiple criteria decision making methods for healthcare management in West China Hospital
}

\author{
Huchang Liao ${ }^{1}$, Cheng Zhang ${ }^{1, *}$, Li Luo ${ }^{1}$, Zeshui Xu${ }^{1}$, Jian-Bo Yang ${ }^{2}$, Dong-Ling Xu² \\ ${ }^{1}$ Business School, Sichuan University, Chengdu 610064, China \\ ${ }^{2}$ Alliance Manchester Business School, The University of Manchester, M15 6PB Manchester, UK
}

\begin{abstract}
Intuitionistic multiplicative sets use an asymmetric, unbalanced scale to express information from positive, negative and indeterminate information. They have been found capable of comprehensively and objectively representing a person's intuitive understanding, and hence have attracted much attention. Distance techniques are widely used to measure the degree to which arguments deviate from one another. Several fuzzy set extensions have been developed, but little research has been conducted on measures of distance between intuitionistic multiplicative sets. In this paper we start by presenting a variety of measures of the distance between intuitionistic multiplicative sets, including Hausdorff distance measures, weighted distance measures, ordered weighted distance measures and continuous weighted distance measures. We then develop a distance-based IM-TOPSIS method and a distancebased IM-VIKOR method for handling multiple criteria decision making problems with intuitionistic multiplicative evaluation information. To demonstrate the practical application of these distance measures and the proposed methods, we provide a case study of hospital management of inpatient admission. The paper ends with comparative analyses of the two methods and some concluding remarks.
\end{abstract}

Keywords: Multiple criteria decision making; intuitionistic multiplicative sets; distance measures; hospital management; inpatient admission

\section{Introduction}

Hospitals are complex systems, always involving various complex information and processes and the issue of

\footnotetext{
*Corresponding author. E-mail addresses: liaohuchang@163.com (H.C. Liao), z_zhangcheng_c@163.com (C. Zhang). 
finding ways to manage them effectively is highly important. The imbalance between supply and demand for scarce medical resources is increasing in China with a huge population pressure. Because the service provided by primary medical and health institutions tends to be poor, patients in China prefer to go to large public hospitals than small ones, regardless how serious their illness is (Zhang et al., 2016). In the case of patients with severe diseases or acute medical problems, a delay of treatment may lead to a high risk of disease progression or death, but patients with minor ailments should be directed to community hospitals or community clinics for treatment. China has many more primary health care institutions than large public hospitals, so the Chinese government is trying to establish a hierarchical system of healthcare triage, which not only helps patients select the most appropriate institution but also helps hospital administrators determine which patients should be treated first. The patient admission process is very important in improving the efficiency with which medical resources are used, especially in large public hospitals such as the West China Hospital (WCH).

Unfortunately, the task of ranking patients is a difficult and subjective one, and an efficient, scientific decision support techniques are needed to aid the process. More importantly, for the patient admission evaluation, the intricacies of objective matters mean it is difficult for people to describe their opinions accurately, especially in the DMs' opinions on qualitative criteria. Fuzzy set (FS) theory provides a tool for investigating such problems. It mainly uses a certain value for the interval $[0,1]$, called the membership degree, to represent the degree to which an element belongs to a set. Through deeper research, scholars have found that it is difficult to determine the exact membership degree for the traditional FS. Atanassov (1986) proposed the use of the intuitionistic FS (IFS) to solve this problem; the IFS is characterized by a membership degree, a non-membership degree and a hesitancy degree, to represent people's cognition from three different aspects. The use of the IFS has led to great achievements in the past three decades since 1986 (Liu \& Liao, 2017; Yu \& Liao, 2018), but IFSs can only represent people's cognition symmetrically by using a balanced scale, i.e., 0.1-0.9 scale, whilst in many practical cases, the preferences of experts or decision-makers (DMs) are actually uniformly and symmetrically distributed. One simple example comes from the law of diminishing marginal utility in economics (Xia, Xu \& Liao, 2013): if inefficient and efficient companies are given the same resources, the growth rate of the latter should be much higher. It is noted that Saaty (1994) proposed the asymmetric 1/9-9 scale, which can be used to describe asymmetric problems. Xia, $\mathrm{Xu}$ and Liao (2013) combined the asymmetric 1/9-9 scale with the concept of the IFS, and introduced the intuitionistic multiplicative preference relation (IMPR) and the concept of intuitionistic multiplicative sets (IMSs). 
IMSs use an asymmetric, unbalanced scale to express three different aspects of information, and thus can provide a comprehensive, objective representation of intuitive information. A lot of work has been done with IMSs (Xu, 2013; Jiang, Xu \& Gao, 2015; Jiang, Xu \& Yu, 2015; Jiang, Xu \& Shu, 2016; Ren, Xu \& Liao, 2016; Zhang \& Pedrycz, 2017, 2018; Luo, Zhang, \& Liao, 2019; Zhang, Liao, \& Luo, 2019). As the fundamental theory for investigating the IMS, distance measure for IMS plays a significant role in measuring the difference between numbers (points or sets). Meanwhile, it is also the core part for most multiple criteria decision making (MCDM) methods, such as the TOPSIS (Technique for Order Preference by Similarity to Ideal Solution) and VIKOR (Vlsekriterijumska Optimizacija I Kompromisno Resenje in Serbian).

So far, distance measures can be classified into traditional distance measures (such as the Hamming distance, the Euclidean distance, the Hausdorff distance) and weighted distance measures (including ordered weighted distance (Xu \& Chen, 2008), continued weighted distance (Xu, 2012), and hybrid weighted distance (Xu, 2008)). Many scholars have investigated distance measures for different kinds of fuzzy information, such as IFSs (Burillo \& Bustince, 1996; Du \& Hu, 2015; Grzegorzewski, 2004; Grzegorzewski, 2004; Narukawa \& Torra, 2006; Szmidt \& Kacprzyk, 2000; Wang \& Xin, 2005; Xu \& Chen, 2011), hesitant fuzzy sets (HFSs) (Xu \& Xia, 2011), and hesitant fuzzy linguistic term sets (HFLTSs) (Liao, Xu \& Zeng, 2014; Liao \& Xu, 2015). As we can see, there have been many advances in approaches to measuring the distance between IFSs but little research on measuring the distance between IMSs (Jiang, Xu \& Gao, 2015; Jiang, Xu \& Shu, 2016; Garg, 2017). Thus, in this paper we focus on measures of the distance between IMSs, which are basic and highly important, especially in solving MCDM problems with IMSs.

In addition, many scholars have investigated this issue regarding the patient admission evaluation, but there are drawbacks to all the approaches proposed so far. First, the approaches proposed for handing inpatient admission management in other countries (see Ashour \& Kremer, 2016; Mariotti, 2014; Rahimi, 2015; Rahimi et al., 2016; Solans-Domènech et al., 2013; Valente et al., 2009) are not appropriate to the China context. Second, although some Chinese scholars (Zhang et al., 2016) have considered the problem they have only considered evaluation criteria such as "urgency", "need for hospitalization", "value of clinical pathology" and not other factors such as the limitations on the person's activity or time spent on the waiting list. Here we present a case study of inpatient admission management by the admission center of the WCH to illustrate how distance-based MCDM methods with IMSs can be used to circumvent these drawbacks by combining with the specific situations of Chinese hospital. 
Inspired by these motivations, in this paper, we investigate the Hausdorff distance measure for IMSs associated with its hybrid form, generalized weighted form, ordered weighted form and continuous weighted form. Comparing with the existing distance measures, including the Minkowski distance, Hamming distance, Euclidean distance (Jiang, Xu \& Gao, 2015; Jiang, Xu \& Shu, 2016; Garg, 2017), the Hausdorff distance has good noise immunity, especially in pattern recognition, image matching and distinct improved algorithms. Moreover, both the ordered weighted form and the continuous form are analyzed in this paper, by which the DMs can select the optimal one according to their demands. Subsequently, based on these novel distance measures between IMSs, we investigate a distance-based IM-TOPSIS method and a distance-based IM-VIKOR method. Both of these two methods can be used to solve MCDM problems with IMS information. Finally, we apply the proposed methods to tackle the problem related to inpatients admission evaluation, which can provide some insights for hospital management in China.

In summary, the contributions of this paper are summarized as follows:

(1) We propose a variety of distance measures for IMSs, based on traditional distance measures such as the Hausdorff distance measures, hybrid distance measures, weighted distance measures, ordered weighted distance measures and weighted distance measures in continuous form. We also provide some numerical illustrations.

(2) We present a distance-based IM-TOPSIS method and a distance-based IM-VIKOR method. These two methods have wide applicability in solving MCDM problems with IMSs. It should be noted that any type of distance measure could be applied in these two methods, so they provide DMs a wider choice for solving MCDM problems.

(3) We apply the proposed distance-based IM-TOPSIS method and distance-based IM-VIKOR method to inpatient admission assessment to aid the WCH admission center. These methods can be used to select patients scientifically and are useful for allocating medical resources in a rational way.

The remainder of this paper is organized as follows: In Section 2 we review some concepts relevant to IMSs as well as the existing measures of distance between IMSs. Section 3 presents various measures of the distance between IMSs. Section 4 investigates the IM-TOPSIS method and IM-VIKOR method based on measures of the distance between IMSs. A case study of application of the IM-TOPSIS and IM-VIKOR methods to inpatient admission assessment is presented in Section 5. Section 6 provides some concluding remarks. 


\section{IMSs and their existing distance measures}

To start our study, we first present the existing distance measures of IMSs.

For a reference set $X$, an IMS on $X$ can be defined as (Xia, Xu \& Liao, 2013):

$$
D=\left\{<x,\left(\rho_{D}(x), \sigma_{D}(x)\right)>\mid x \in X\right\}
$$

where $\rho_{D}(x)$ and $\sigma_{D}(x)$ are the membership degree the non-membership degree of $x \in X$ to the set $D$, respectively, and they satisfy: $1 / 9 \leq \rho_{D}(x), \sigma_{D}(x) \leq 9, \rho_{D}(x) \sigma_{D}(x) \leq 1, \forall x \in X . \tau_{D}(x)=1 / \rho_{D}(x) \sigma_{D}(x)$ is called the hesitancy degree and $\left(\rho_{D}(x), \sigma_{D}(x)\right)$ is called an IMN. For any two IMNs, they can be compared by the score function $s(\alpha)=\rho_{\alpha} / \sigma_{\alpha}$ and the accuracy function $h(\alpha)=\rho_{\alpha} \sigma_{\alpha}$.

Assume that an DM evaluates two alternatives $x_{i}$ and $x_{j}$ on a criterion and gives his/her opinions as IMS $\left(\rho_{D}(x), \sigma_{D}(x)\right)$. For an IMS $\left(\rho_{D}(x), \sigma_{D}(x)\right)$, the term $\rho_{D}(x) \in\left[\frac{1}{9}, 1\right)$ denotes that the alternative $x_{i}$ is inferior to $x_{j}$, that is, the alternative $x_{j}$ is prior to $x_{i}$. In contrast, the term $\rho_{D}(x) \in(1,9]$ means the alternative $x_{i}$ is preferred to $x_{j}$. In addition, several special cases can be described: (1) $\rho_{D}(x)=\sigma_{D}(x)=1$ represents that there is no difference between $x_{i}$ and $x_{j} ;$ (2) $\rho_{D}(x)=9$ or $\sigma_{D}(x)=\frac{1}{9}$ indicates that $x_{i}$ is absolutely preferred to $x_{j} ;(3)$ $\rho_{D}(x)=\frac{1}{9}$ or $\sigma_{D}(x)=9$ denotes that $x_{i}$ is absolutely inferior to $x_{j}$. For details, please refer to Xia, Xu and Liao (2013).

For two IMSs $A=\left\{\left(\rho_{A}\left(x_{i}\right), \sigma_{A}\left(x_{i}\right), \tau_{A}\left(x_{i}\right)\right) \mid x_{i} \in X\right\}$ and $B=\left\{\left(\rho_{B}\left(x_{i}\right), \sigma_{B}\left(x_{i}\right), \tau_{B}\left(x_{i}\right)\right) \mid x_{i} \in X\right\}$, Jiang, Xu \& Gao (2015) proposed that the distance measure $d(A, B)$ should satisfy the following basic axioms: (1) $0 \leq d(A, B) \leq 1$; (2) $d(A, B)=0$ if and only if $A=B$; (3) $d(A, B)=d(B, A)$; (4) For $A, B, C \in M$, if $A \subseteq B \subseteq C$, then $d(A, C) \geq d(A, B)$ and $d(A, C) \geq d(B, C)$. Then, they defined the Minkowski distance measure and the normalized Minkowski distance measure between IMSs $A$ and $B$ as:

$$
d_{m d}(A, B)=\left(\frac{1}{2^{\lambda+1}} \sum_{i=1}^{n}\left(\left|\log _{9} \frac{\rho_{A}\left(x_{i}\right)}{\rho_{B}\left(x_{i}\right)}\right|^{\lambda}+\left|\log _{9} \frac{\sigma_{A}\left(x_{i}\right)}{\sigma_{B}\left(x_{i}\right)}\right|^{\lambda}+\left|\log _{9} \frac{\tau_{A}\left(x_{i}\right)}{\tau_{B}\left(x_{i}\right)}\right|^{\lambda}\right)\right)^{1 / \lambda}, \lambda \geq 1
$$




$$
d_{n m d}(A, B)=\left(\frac{1}{2^{\lambda+1} n} \sum_{i=1}^{n}\left(\left|\log _{9} \frac{\rho_{A}\left(x_{i}\right)}{\rho_{B}\left(x_{i}\right)}\right|^{\lambda}+\left|\log _{9} \frac{\sigma_{A}\left(x_{i}\right)}{\sigma_{B}\left(x_{i}\right)}\right|^{\lambda}+\left|\log _{9} \frac{\tau_{A}\left(x_{i}\right)}{\tau_{B}\left(x_{i}\right)}\right|^{\lambda}\right)\right)^{1 / \lambda}, \lambda \geq 1
$$

If $\lambda=1$, the Minkowski distance becomes the Hamming distance and the normalized Minkowski distance becomes the normalized Hamming distance; If $\lambda=2$, the Minkowski distance becomes the Euclidean distance and the normalized Minkowski distance becomes the normalized Euclidean distance (Jiang, Xu \& Shu, 2016).

Theorem 1. For two IMSs $A$ and $B$, if $\lambda \rightarrow+\infty$, the normalized Minkowski distance measure reduces to the following:

$$
\lim _{\lambda \rightarrow+\infty} d_{n m d}(A, B)=\frac{1}{2} \max _{i}\left\{\left|\log _{9} \frac{\rho_{A}\left(x_{i}\right)}{\rho_{B}\left(x_{i}\right)}\right|,\left|\log _{9} \frac{\sigma_{A}\left(x_{i}\right)}{\sigma_{B}\left(x_{i}\right)}\right|,\left|\log _{9} \frac{\tau_{A}\left(x_{i}\right)}{\tau_{B}\left(x_{i}\right)}\right|\right\}
$$

Proof: Let $\alpha=\frac{1}{2} \max _{i}\left\{\left|\log _{9} \frac{\rho_{A}\left(x_{i}\right)}{\rho_{B}\left(x_{i}\right)}\right|,\left|\log _{9} \frac{\sigma_{A}\left(x_{i}\right)}{\sigma_{B}\left(x_{i}\right)}\right|,\left|\log _{9} \frac{\tau_{A}\left(x_{i}\right)}{\tau_{B}\left(x_{i}\right)}\right|\right\}$. Then by Eq. (3), we have

$\lim _{\lambda \rightarrow+\infty} d_{n m d}(A, B)=\lim _{\lambda \rightarrow+\infty}\left(\frac{1}{2^{\lambda+1} n} \sum_{i=1}^{n}\left(\left|\log _{9} \frac{\rho_{A}\left(x_{i}\right)}{\rho_{B}\left(x_{i}\right)}\right|^{\lambda}+\left|\log _{9} \frac{\sigma_{A}\left(x_{i}\right)}{\sigma_{B}\left(x_{i}\right)}\right|^{\lambda}+\left|\log _{9} \frac{\tau_{A}\left(x_{i}\right)}{\tau_{B}\left(x_{i}\right)}\right|^{\lambda}\right)\right)^{1 / \lambda}$

$=\lim _{\lambda \rightarrow+\infty} \alpha\left[\frac{1}{2^{\lambda+1} n} \sum_{i=1}^{n}\left(\left|\log _{9} \frac{\rho_{A}\left(x_{i}\right)}{\rho_{B}\left(x_{i}\right)}\right| / \alpha\right)^{\lambda}+\left(\left|\log _{9} \frac{\sigma_{A}\left(x_{i}\right)}{\sigma_{B}\left(x_{i}\right)}\right| / \alpha\right)^{\lambda}+\left(\left|\log _{9} \frac{\tau_{A}\left(x_{i}\right)}{\tau_{B}\left(x_{i}\right)}\right| / \alpha\right)^{\lambda}\right]^{1 / \lambda}$

$=\alpha \lim _{\lambda \rightarrow+\infty}\left(\frac{1}{2 n}\right)^{\frac{1}{\lambda}} \cdot \lim _{\lambda \rightarrow+\infty}\left[\sum_{i=1}^{n}\left(\left(\left|\frac{1}{2} \log _{9} \frac{\rho_{A}\left(x_{i}\right)}{\rho_{B}\left(x_{i}\right)}\right| / \alpha\right)^{\lambda}+\left(\left|\frac{1}{2} \log _{9} \frac{\sigma_{A}\left(x_{i}\right)}{\sigma_{B}\left(x_{i}\right)}\right| / \alpha\right)^{\lambda}+\left(\left|\frac{1}{2} \log _{9} \frac{\tau_{A}\left(x_{i}\right)}{\tau_{B}\left(x_{i}\right)}\right| / \alpha\right)^{\lambda}\right)\right]^{1 / \lambda}=\alpha$

This completes the proof of Theorem 1.

\section{Hausdorff metric-based distance measures for IMSs}

This section introduces new distance measures between IMSs.

\subsection{Hausdorff distance measures and hybrid distance measures between IMSs}

Jiang, Xu and Gao (2015), Jiang, Xu and Shu (2016) and Garg (2017) introduced a series of distance measures for IMSs, including the Manhattan distance, the Euclidean distance, and the Minkowski distance, together with the corresponding normalized and weighted forms. However, the Euclidean distance, one of the most widely used distance measures, fails to measure the difference between different types of attribute. We know that different types 
of attribute have different dimensions and the Euclidean distance treats them equally, such that the results are inconsistent with reality. The Hausdorff distance, a new definition of distance, not only overcomes the defect of Euclidean distance but also has good noise immunity and so it has been widely applied to pattern recognition, image matching and distinct improved algorithms (Du \& Hu, 2015; Ashour \& Kremer, 2016). The application of the Hausdorff distance to various information forms, such as IFSs (Grzegorzewski, 2004), HFSs (Xu \& Xia, 2011) and HFLTSs (Liao, Xu \& Zeng, 2014) has also been investigated. However, there has been little research into use of the Hausdorff distance measure with IMSs. We therefore introduce some measures of the distance between IMSs based on the Hausdorff metric.

Inspired by the generalized Hausdorff measures of the distance between IFSs (Grzegorzewski, 2004) and taking into account the relationships between IMSs and IFSs, we can define a generalized Hausdorff measure of the distance between two IMSs $A$ and $B$ as:

$$
d_{\text {ghaud }}(A, B)=\left(\frac{1}{2^{\lambda}} \sum_{i=1}^{n} \max \left\{\left|\log _{9} \frac{\rho_{A}\left(x_{i}\right)}{\rho_{B}\left(x_{i}\right)}\right|^{\lambda},\left|\log _{9} \frac{\sigma_{A}\left(x_{i}\right)}{\sigma_{B}\left(x_{i}\right)}\right|^{\lambda},\left|\log _{9} \frac{\tau_{A}\left(x_{i}\right)}{\tau_{B}\left(x_{i}\right)}\right|^{\lambda}\right\}\right)^{1 / \lambda}, \lambda>0
$$

Property 1. The generalized Hausdorff distance measure shown as Eq. (5) satisfies the following properties:

(1) $d_{\text {ghaud }}(A, B)=0$ if and only if $A=B$;

(2) $d_{\text {ghaud }}(A, B)=d_{\text {ghaud }}(B, A)$;

(3) If $A \subseteq B \subseteq C$, then $d_{\text {ghaud }}(A, B)+d_{\text {ghaud }}(B, C) \geq d_{\text {ghaud }}(A, C)$.

The proof of Property 1 is given in the Appendix.

If $\lambda=1$, then the generalized Hausdorff distance becomes the Hamming-Hausdorff distance:

$$
d_{\text {hhaud }}(A, B)=\frac{1}{2} \sum_{i=1}^{n} \max \left\{\left|\log _{9} \frac{\rho_{A}\left(x_{i}\right)}{\rho_{B}\left(x_{i}\right)}\right|,\left|\log _{9} \frac{\sigma_{A}\left(x_{i}\right)}{\sigma_{B}\left(x_{i}\right)}\right|,\left|\log _{9} \frac{\tau_{A}\left(x_{i}\right)}{\tau_{B}\left(x_{i}\right)}\right|\right\}
$$

If $\lambda=2$, then the generalized Hausdorff distance becomes the Euclidean-Hausdorff distance:

$$
d_{\text {ehaud }}(A, B)=\sqrt{\frac{1}{4} \sum_{i=1}^{n} \max \left\{\left|\log _{9} \frac{\rho_{A}\left(x_{i}\right)}{\rho_{B}\left(x_{i}\right)}\right|^{2},\left|\log _{9} \frac{\sigma_{A}\left(x_{i}\right)}{\sigma_{B}\left(x_{i}\right)}\right|^{2},\left|\log _{9} \frac{\tau_{A}\left(x_{i}\right)}{\tau_{B}\left(x_{i}\right)}\right|^{2}\right\}}
$$

In addition, if we combine the Hausdorff distance with the Hamming distance and the Euclidean distance, we can obtain some hybrid measures of the distance between $A$ and $B$, such as the hybrid Hamming distance, the hybrid Euclidean distance and the generalized hybrid distance, which are shown below in that order: 


$$
\begin{gathered}
d_{\text {hhd }}(A, B)=\sum_{i=1}^{n}\left(\frac{1}{8}\left(\left|\log _{9} \frac{\rho_{A}\left(x_{i}\right)}{\rho_{B}\left(x_{i}\right)}\right|+\left|\log _{9} \frac{\sigma_{A}\left(x_{i}\right)}{\sigma_{B}\left(x_{i}\right)}\right|+\left|\log _{9} \frac{\tau_{A}\left(x_{i}\right)}{\tau_{B}\left(x_{i}\right)}\right|\right)+\frac{1}{4} \max \left\{\left|\log _{9} \frac{\rho_{A}\left(x_{i}\right)}{\rho_{B}\left(x_{i}\right)}\right|,\left|\log _{9} \frac{\sigma_{A}\left(x_{i}\right)}{\sigma_{B}\left(x_{i}\right)}\right|,\left|\log _{9} \frac{\tau_{A}\left(x_{i}\right)}{\tau_{B}\left(x_{i}\right)}\right|\right\}\right) \\
d_{\text {hed }}(A, B)=\left(\sum_{i=1}^{n}\left(\frac{1}{16}\left(\left|\log _{9} \frac{\rho_{A}\left(x_{i}\right)}{\rho_{B}\left(x_{i}\right)}\right|^{2}+\left|\log _{9} \frac{\sigma_{A}\left(x_{i}\right)}{\sigma_{B}\left(x_{i}\right)}\right|^{2}+\left|\log _{9} \frac{\tau_{A}\left(x_{i}\right)}{\tau_{B}\left(x_{i}\right)}\right|^{2} \mid+\frac{1}{8} \max \left\{\left|\log _{9} \frac{\rho_{A}\left(x_{i}\right)}{\rho_{B}\left(x_{i}\right)}\right|^{2},\left|\log _{9} \frac{\sigma_{A}\left(x_{i}\right)}{\sigma_{B}\left(x_{i}\right)}\right|^{2},\left|\log _{9} \frac{\tau_{A}\left(x_{i}\right)}{\tau_{B}\left(x_{i}\right)}\right|\right\}\right)\right)^{1 / 2}\right. \\
d_{g h d}(A, B)=\left(\sum_{i=1}^{n}\left(\frac{1}{2^{\lambda+2}}\left(\left|\log _{9} \frac{\rho_{A}\left(x_{i}\right)}{\rho_{B}\left(x_{i}\right)}\right|^{\lambda}+\left|\log _{9} \frac{\sigma_{A}\left(x_{i}\right)}{\sigma_{B}\left(x_{i}\right)}\right|^{\lambda}+\left|\log _{9} \frac{\tau_{A}\left(x_{i}\right)}{\tau_{B}\left(x_{i}\right)}\right|^{\lambda} \mid+\frac{1}{2^{\lambda+1}} \max \left\{\left|\log _{9} \frac{\rho_{A}\left(x_{i}\right)}{\rho_{B}\left(x_{i}\right)}\right|^{\lambda},\left|\log _{9} \frac{\sigma_{A}\left(x_{i}\right)}{\sigma_{B}\left(x_{i}\right)}\right|^{\lambda},\left|\log _{9} \frac{\tau_{A}\left(x_{i}\right)}{\tau_{B}\left(x_{i}\right)}\right|^{\lambda}\right\}\right)\right)^{1 / \lambda}, \lambda>0(\right.
\end{gathered}
$$

Property 2. The generalized hybrid distance measure shown as Eq. (10) satisfies the following properties:

(1) $d_{g h d}(A, B)=0$ if and only if $A=B$;

(2) $d_{g h d}(A, B)=d_{g h d}(B, A)$;

(3) If $A \subseteq B \subseteq C$, then $d_{g h d}(A, B)+d_{g h d}(B, C) \geq d_{g h d}(A, C)$.

The proof of Property 2 is given in the Appendix.

\subsection{Distinct weighted distance measures between IMSs}

In this subsection, we mainly introduce the distance measures between IMSs from three aspects, which are the weighted distance measures, the ordered weighted distance measures, and the continuously weighted distance measures.

\subsubsection{Weighted distance measures between IMSs}

In many real-world situations the weights of the elements $x_{i} \in X(i=1,2, \cdots, n)$ should be considered. Jiang, $\mathrm{Xu}$ and Shu (2016) proposed some weighted measures of the distance between IMSs, such as the weighted Minkowski distance, the weighted Hamming distance, and the weighted Euclidean distance. In addition, Garg (2017) presented the normalized weighted Hamming distance and the normalized weighted Euclidean distance. Below we introduce more weighted distance measures for IMSs.

Let $X=\left\{x_{1}, x_{2}, \cdots, x_{n}\right\} \quad$ be $\quad$ a $\quad$ finite $\quad$ set, $\left.\quad A=\left\{\rho_{A}\left(x_{i}\right), \quad \sigma_{A}\left(x_{i}\right), \tau\left(x_{i}\right)\right) \mid x_{i} \in X\right\} \quad$ and $\quad B=$ $\left\{\left(\rho_{B}\left(x_{i}\right), \sigma_{B}\left(x_{i}\right), \tau_{B}\left(x_{i}\right)\right) \mid x_{i} \in X\right\}$ be two IMSs in $X$ associated with the weight vector $w=\left(w_{1}, w_{2}, \cdots, w_{n}\right)^{T}$, where $0 \leq w_{i} \leq 1$ and $\sum_{i=1}^{n} w_{i}=1$. The generalized weighted Hausdorff distance between $A$ and $B$ is defined as: 


$$
d_{\text {gwhaud }}(A, B)=\left(\frac{1}{2^{\lambda}} \sum_{i=1}^{n} w_{i} \max \left\{\left|\log _{9} \frac{\rho_{A}\left(x_{i}\right)}{\rho_{B}\left(x_{i}\right)}\right|^{\lambda},\left|\log _{9} \frac{\sigma_{A}\left(x_{i}\right)}{\sigma_{B}\left(x_{i}\right)}\right|^{\lambda},\left|\log _{9} \frac{\tau_{A}\left(x_{i}\right)}{\tau_{B}\left(x_{i}\right)}\right|^{\lambda}\right\}\right)^{1 / \lambda}, \lambda>0
$$

If $\lambda=1$, we can obtain the weighted Hamming-Hausdorff distance between $A$ and $B$ :

$$
\left.d_{\text {whhaud }}(A, B)=\frac{1}{2} \sum_{i=1}^{n} w_{i} \max \left\{\left|\log _{9} \frac{\rho_{A}\left(x_{i}\right)}{\rho_{B}\left(x_{i}\right)}\right|, \mid \log _{9} \frac{\sigma_{A}\left(x_{i}\right)}{\sigma_{B}\left(x_{i}\right)}\right)\left|, \log _{9} \frac{\tau_{A}\left(x_{i}\right)}{\tau_{B}\left(x_{i}\right)}\right|\right\}
$$

If $\lambda=2$, we can obtain the weighted Euclidean-Hausdorff distance between $A$ and $B$ :

$$
d_{\text {wehaud }}(A, B)=\sqrt{\frac{1}{4} \sum_{i=1}^{n} w_{i} \max \left\{\left|\log _{9} \frac{\rho_{A}\left(x_{i}\right)}{\rho_{B}\left(x_{i}\right)}\right|^{2},\left|\log _{9} \frac{\sigma_{A}\left(x_{i}\right)}{\sigma_{B}\left(x_{i}\right)}\right|^{2},\left|\log _{9} \frac{\tau_{A}\left(x_{i}\right)}{\tau_{B}\left(x_{i}\right)}\right|^{2}\right\}}
$$

In particular, if $w=(1 / n, 1 / n, \cdots, 1 / n)^{T}$, then the generalized weighted distance, the weighted Hamming distance, and the weighted Euclidean distance are equal to the normalized Minkowski distance, the normalized Hamming distance and the normalized Euclidean distance, respectively.

Obviously, we can also obtain some hybrid weighted distance measures on the basis of the above distance measures, for example:

(1) The hybrid weighted Hamming distance between $A$ and $B$ :

$$
\left.d_{\text {hwhd }}(A, B)=\sum_{i=1}^{n} w_{i}\left[\frac{1}{8}\left(\left|\log _{9} \frac{\rho_{A}\left(x_{i}\right)}{\rho_{B}\left(x_{i}\right)}\right|+\left|\log _{9} \frac{\sigma_{A}\left(x_{i}\right)}{\sigma_{B}\left(x_{i}\right)}\right|+\left|\log _{9} \frac{\tau_{A}\left(x_{i}\right)}{\tau_{B}\left(x_{i}\right)}\right|\right)+\frac{1}{4} \max \left\{\left|\log _{9} \frac{\rho_{A}\left(x_{i}\right)}{\rho_{B}\left(x_{i}\right)}\right|, \mid \log _{9} \frac{\sigma_{A}\left(x_{i}\right)}{\sigma_{B}\left(x_{i}\right)}\right)|| \log _{9} \frac{\tau_{A}\left(x_{i}\right)}{\tau_{B}\left(x_{i}\right)} \mid\right\}\right]
$$

(2) The hybrid weighted Euclidean distance between $A$ and $B$ :

$$
d_{\text {hwed }}(A, B)=\left[\sum_{i=1}^{n} w_{i}\left(\frac{1}{16}\left(\left|\log _{9} \frac{\rho_{A}\left(x_{i}\right)}{\rho_{B}\left(x_{i}\right)}\right|^{2}+\left|\log _{9} \frac{\sigma_{A}\left(x_{i}\right)}{\sigma_{B}\left(x_{i}\right)}\right|^{2}+\left|\log _{9} \frac{\tau_{A}\left(x_{i}\right)}{\tau_{B}\left(x_{i}\right)}\right|^{2}\right)+\frac{1}{8} \max \left\{\left|\log _{9} \frac{\rho_{A}\left(x_{i}\right)}{\rho_{B}\left(x_{i}\right)}\right|^{2}\left|, \log _{9} \frac{\sigma_{A}\left(x_{i}\right)}{\sigma_{B}\left(x_{i}\right)}\right|^{2}\left|\log _{9} \frac{\tau_{A}\left(x_{i}\right)}{\tau_{B}\left(x_{i}\right)}\right|^{2}\right\}\right]^{1 / 2}\right.
$$

(3) The generalized hybrid weighted distance between $A$ and $B$ :

$$
d_{g h w d}(A, B)=\left[\sum_{i=1}^{n} w_{i}\left(\frac{1}{2^{\lambda+2}}\left(\left|\log _{9} \frac{\rho_{A}\left(x_{i}\right)}{\rho_{B}\left(x_{i}\right)}\right|^{2}+\left|\log _{9} \frac{\sigma_{A}\left(x_{i}\right)}{\sigma_{B}\left(x_{i}\right)}\right|^{2}+\left|\log _{9} \frac{\tau_{A}\left(x_{i}\right)}{\tau_{B}\left(x_{i}\right)}\right|^{\lambda}\right)+\frac{1}{2^{\lambda+1}} \max \left\{\left|\log _{9} \frac{\rho_{A}\left(x_{i}\right)}{\rho_{B}\left(x_{i}\right)}\right|^{2},\left|\log _{9} \frac{\sigma_{A}\left(x_{i}\right)}{\sigma_{B}\left(x_{i}\right)}\right|^{2}\left|\log _{9} \frac{\tau_{A}\left(x_{i}\right)}{\tau_{B}\left(x_{i}\right)}\right|\right\}\right)\right]^{1 / \lambda}, \lambda>0
$$

\subsubsection{Ordered weighted measures of the distance between IMSs}

The concept of ordered weighted distance was introduced by $\mathrm{Xu}$ and Chen (2008) and has been studied by many scholars. So far the concept of ordered weighted distance has been extended to IFSs (Xu \& Chen, 2011), HFSs (Xu \& Xia, 2011) and HFLTSs (Liao \& Xu, 2015; Liao, Xu \& Zeng, 2014). A prominent characteristic of ordered weighted distance is that it can diminish or enhance the influence of unduly large or small deviations on the 
aggregation results by assigning them low or high weights (Xu \& Chen, 2008). This desirable property makes ordered weighted distance measures extremely useful in decision making processes, however our review of the existing literature revealed that there has been no research on ordered weighted measures of the distance between IMSs, so in this subsection we present some ordered weighted measures of the distance between IMSs.

Let $A$ and $B$ be two IMSs, and their associated weight vector be given as above. The generalized ordered weighted distance between $A$ and $B$ is defined as:

$$
d_{\text {gowd }}(A, B)=\left[\frac{1}{2^{\lambda+1}} \sum_{i=1}^{n} w_{i}\left(\left|\log _{9} \frac{\rho_{A}\left(x_{\eta(i)}\right)}{\rho_{B}\left(x_{\eta(i)}\right)}\right|^{\lambda}+\left|\log _{9} \frac{\sigma_{A}\left(x_{\eta(i)}\right)}{\sigma_{B}\left(x_{\eta(i)}\right)}\right|^{\lambda}+\left|\log _{9} \frac{\tau_{A}\left(x_{\eta(i)}\right)}{\tau_{B}\left(x_{\eta(i)}\right)}\right|^{\lambda}\right)\right]^{1 / \lambda}, \lambda>0
$$

where $\eta(1), \eta(2), \cdots, \eta(n)$ is a permutation of $(1,2, \cdots, n)$ such that

$$
\left(\left|\log _{9} \frac{\rho_{A}\left(x_{\eta(i-1)}\right)}{\rho_{B}\left(x_{\eta(i-1)}\right)}\right|^{\lambda}+\left|\log _{9} \frac{\sigma_{A}\left(x_{\eta(i-1)}\right)}{\sigma_{B}\left(x_{\eta(i-1)}\right)}\right|^{\lambda}+\left|\log _{9} \frac{\tau_{A}\left(x_{\eta(i-1)}\right)}{\tau_{B}\left(x_{\eta(i-1)}\right)}\right|^{\lambda}\right) \geq\left(\left|\log _{9} \frac{\rho_{A}\left(x_{\eta(i)}\right)}{\rho_{B}\left(x_{\eta(i)}\right)}\right|^{\lambda}+\left|\log _{9} \frac{\sigma_{A}\left(x_{\eta(i)}\right)}{\sigma_{B}\left(x_{\eta(i)}\right)}\right|^{\lambda}+\left|\log _{9} \frac{\tau_{A}\left(x_{\eta(i)}\right)}{\tau_{B}\left(x_{\eta(i)}\right)}\right|^{\lambda}\right)
$$

If $\lambda=1$, Eq. (17) reduces to an ordered weighted Hamming distance between $A$ and $B$ :

$$
d_{\text {owhd }}(A, B)=\frac{1}{4} \sum_{i=1}^{n} w_{i}\left(\left|\log _{9} \frac{\rho_{A}\left(x_{\eta(i)}\right)}{\rho_{B}\left(x_{\eta(i)}\right)}\right|+\left|\log _{9} \frac{\sigma_{A}\left(x_{\eta(i)}\right)}{\sigma_{B}\left(x_{\eta(i)}\right)}\right|+\left|\log _{9} \frac{\tau_{A}\left(x_{\eta(i)}\right)}{\tau_{B}\left(x_{\eta(i)}\right)}\right|\right)
$$

If $\lambda=2$, Eq. (17) reduces to an ordered weighted Euclidean distance between $A$ and $B$ :

$$
d_{\text {owed }}(A, B)=\sqrt{\frac{1}{8} \sum_{i=1}^{n} w_{i}\left(\left|\log _{9} \frac{\rho_{A}\left(x_{\eta(i)}\right)}{\rho_{B}\left(x_{\eta(i)}\right)}\right|^{2}+\left|\log _{9} \frac{\sigma_{A}\left(x_{\eta(i)}\right)}{\sigma_{B}\left(x_{\eta(i)}\right)}\right|^{2}+\left|\log _{9} \frac{\tau_{A}\left(x_{\eta(i)}\right)}{\tau_{B}\left(x_{\eta(i)}\right)}\right|^{2}\right.}
$$

Based on the Hausdorff metric, we can define a generalized ordered weighted Hausdorff distance between $A$ and $B$ :

$$
d_{\text {gowhaud }}(A, B)=\left[\frac{1}{2^{\lambda}} \sum_{i=1}^{n} w_{i} \max \left\{\left.\left|\log _{9} \frac{\rho_{A}\left(x_{\eta(i)}\right)}{\rho_{B}\left(x_{\eta(i)}\right)}\right|^{\lambda}|,| \log _{9} \frac{\sigma_{A}\left(x_{\eta(i)}\right)}{\sigma_{B}\left(x_{\eta(i)}\right)}\right|^{\lambda},\left|\log _{9} \frac{\tau_{A}\left(x_{\eta(i)}\right)}{\tau_{B}\left(x_{\eta(i)}\right)}\right|^{\lambda}\right\}\right]^{1 / \lambda}, \lambda>0
$$

where $\eta(1), \eta(2), \cdots, \eta(n)$ is a permutation of $(1,2, \cdots, n)$, such that:

$$
\max \left\{\left|\log _{9} \frac{\rho_{A}\left(x_{\eta(i)}\right)}{\rho_{B}\left(x_{\eta(i)}\right)}\right|^{\lambda},\left|\log _{9} \frac{\sigma_{A}\left(x_{\eta(i)}\right)}{\sigma_{B}\left(x_{\eta(i)}\right)}\right|^{\lambda},\left|\log _{9} \frac{\tau_{A}\left(x_{\eta(i)}\right)}{\tau_{B}\left(x_{\eta(i)}\right)}\right|^{\lambda}\right\} \geq \max \left\{\left|\log _{9} \frac{\rho_{A}\left(x_{\eta(i+1)}\right)}{\rho_{B}\left(x_{\eta(i+1)}\right)}\right|^{\lambda},\left|\log _{9} \frac{\sigma_{A}\left(x_{\eta(i+1)}\right)}{\sigma_{B}\left(x_{\eta(i+1)}\right)}\right|^{\lambda},\left|\log _{9} \frac{\tau_{A}\left(x_{\eta(i+1)}\right)}{\tau_{B}\left(x_{\eta(i+1)}\right)}\right|^{\lambda}\right\}
$$

If $\lambda=1$, then Eq. (20) reduces to an ordered weighted Hamming-Hausdorff distance between $A$ and $B$ : 


$$
\left.d_{\text {owhhaud }}(A, B)=\frac{1}{2} \sum_{i=1}^{n} w_{i} \max \left\{\left|\log _{9} \frac{\rho_{A}\left(x_{\eta(i)}\right)}{\rho_{B}\left(x_{\eta(i)}\right)}\right|,\left|\log _{9} \frac{\sigma_{A}\left(x_{\eta(i)}\right)}{\sigma_{B}\left(x_{\eta(i)}\right)}\right|, \mid \log _{9} \frac{\tau_{A}\left(x_{\eta(i)}\right)}{\tau_{B}\left(x_{\eta(i)}\right)}\right) \mid\right\}
$$

If $\lambda=2$, then Eq. (20) reduces to an ordered weighted Euclidean-Hausdorff distance between $A$ and $B$ :

$$
d_{\text {owehaud }}(A, B)=\sqrt{\frac{1}{4} \sum_{i=1}^{n} w_{i} \max \left\{\left.\left|\log _{9} \frac{\rho_{A}\left(x_{\eta(i)}\right)}{\rho_{B}\left(x_{\eta(i)}\right)}\right|^{2}|,| \log _{9} \frac{\sigma_{A}\left(x_{\eta(i)}\right)}{\sigma_{B}\left(x_{\eta(i)}\right)}\right|^{2},\left|\log _{9} \frac{\tau_{A}\left(x_{\eta(i)}\right)}{\tau_{B}\left(x_{\eta(i)}\right)}\right|^{2}\right\}}
$$

Obviously, we can also develop some hybrid ordered weighted distance measures, such as the hybrid generalized ordered weighted distance, the hybrid ordered weighted Hamming distance, and the hybrid ordered weighted Euclidean distance between $A$ and $B$, which are shown below in that order:

$$
\begin{aligned}
& d_{\text {ghowd }}(A, B)=\left[\sum_{i=1}^{n} w_{i}\left(\frac{1}{2^{\lambda+2}}\left(\left|\log _{9} \frac{\rho_{A}\left(x_{\eta(i)}\right)}{\rho_{B}\left(x_{\eta(i)}\right)}\right|^{\lambda}+\left|\log _{9} \frac{\sigma_{A}\left(x_{\eta(i)}\right)}{\sigma_{B}\left(x_{\eta(i)}\right)}\right|^{\lambda}+\left|\log _{9} \frac{\tau_{A}\left(x_{\eta(i)}\right)}{\tau_{B}\left(x_{\eta(i)}\right)}\right|^{\lambda}\right)+\frac{1}{2^{\lambda+1}} \max \left\{\left|\log _{9} \frac{\rho_{A}\left(x_{\eta(i)}\right)}{\rho_{B}\left(x_{\eta(i)}\right)}\right|^{\lambda},\left|\log _{9} \frac{\sigma_{A}\left(x_{\eta(i)}\right)}{\sigma_{B}\left(x_{\eta(i)}\right)}\right|^{\lambda},\left|\log _{9} \frac{\tau_{A}\left(x_{\eta(i)}\right)}{\tau_{B}\left(x_{\eta(i)}\right)}\right|^{\lambda}\right)\right\}\right]^{1 / \lambda}, \lambda>0 \\
& \left.d_{\text {howhd }}(A, B)=\sum_{i=1}^{n} w_{i}\left(\frac{1}{8}\left(\left|\log _{9} \frac{\rho_{A}\left(x_{\eta(i)}\right)}{\rho_{B}\left(x_{\eta(i)}\right)}\right|+\left|\log _{9} \frac{\sigma_{A}\left(x_{\eta(i)}\right)}{\sigma_{B}\left(x_{\eta(i)}\right)}\right|+\left|\log _{9} \frac{\tau_{A}\left(x_{\eta(i)}\right)}{\tau_{B}\left(x_{\eta(i)}\right)}\right|\right)+\frac{1}{4} \max \left\{\mid \log _{9} \frac{\rho_{A}\left(x_{\eta(i)}\right)}{\rho_{B}\left(x_{\eta(i)}\right)}\right)\left|\log _{9} \frac{\sigma_{A}\left(x_{\eta(i)}\right)}{\sigma_{B}\left(x_{\eta(i)}\right)}\right|,\left|\log _{9} \frac{\tau_{A}\left(x_{\eta(i)}\right)}{\tau_{B}\left(x_{\eta(i)}\right)}\right|\right\}\right) \\
& d_{\text {hoved }}(A, B)=\left[\sum_{i=1}^{n} w_{i}\left(\frac{1}{16}\left(\left|\log _{9} \frac{\rho_{A}\left(x_{\eta(i)}\right)}{\rho_{B}\left(x_{\eta(i)}\right)}\right|^{2}+\left|\log _{9} \frac{\sigma_{A}\left(x_{\eta(i)}\right)}{\sigma_{B}\left(x_{\eta(i)}\right)}\right|^{2}+\left|\log _{9} \frac{\tau_{A}\left(x_{\eta(i)}\right)}{\tau_{B}\left(x_{\eta(i)}\right)}\right|^{2}\right)+\frac{1}{8} \max \left\{\left|\log _{9} \frac{\rho_{A}\left(x_{\eta(i)}\right)}{\rho_{B}\left(x_{\eta(i)}\right)}\right|^{2},\left|\log _{9} \frac{\sigma_{A}\left(x_{\eta(i)}\right)}{\sigma_{B}\left(x_{\eta(i)}\right)}\right|^{2}, \mid \log _{9} \frac{\tau_{A}\left(x_{\eta(i)}\right)}{\tau_{B}\left(x_{\eta(i)}\right)}\right) \mid\right\}\right]^{1 / 2}
\end{aligned}
$$

where $\eta(1), \eta(2), \cdots, \eta(n)$ is any permutation of $(1,2, \cdots, n)$, such that

$$
\begin{aligned}
& {\left[\frac{1}{16}\left(\left|\log _{9} \frac{\rho_{A}\left(x_{\eta(i)}\right)}{\rho_{B}\left(x_{\eta(i)}\right)}\right|^{\lambda}+\left|\log _{9} \frac{\sigma_{A}\left(x_{\eta(i)}\right)}{\sigma_{B}\left(x_{\eta(i)}\right)}\right|^{\lambda}+\left|\log _{9} \frac{\tau_{A}\left(x_{\eta(i)}\right)}{\tau_{B}\left(x_{\eta(i)}\right)}\right|^{\lambda}\right)+\frac{1}{8} \max \left\{\left|\log _{9} \frac{\rho_{A}\left(x_{\eta(i)}\right)}{\rho_{B}\left(x_{\eta(i)}\right)}\right|^{\lambda},\left|\log _{9} \frac{\sigma_{A}\left(x_{\eta(i)}\right)}{\sigma_{B}\left(x_{\eta(i)}\right)}\right|^{\lambda},\left|\log _{9} \frac{\tau_{A}\left(x_{\eta(i)}\right)}{\tau_{B}\left(x_{\eta(i)}\right)}\right|^{\lambda}\right\} \geq\right.} \\
& {\left[\frac{1}{16}\left(\left|\log _{9} \frac{\rho_{A}\left(x_{\eta(i+1)}\right)}{\rho_{B}\left(x_{\eta(i+1)}\right)}\right|^{\lambda}+\left|\log _{9} \frac{\sigma_{A}\left(x_{\eta(i+1)}\right)}{\sigma_{B}\left(x_{\eta(i+1)}\right)}\right|^{\lambda}+\left|\log _{9} \frac{\tau_{A}\left(x_{\eta(i+1)}\right)}{\tau_{B}\left(x_{\eta(i+1)}\right)}\right|^{\lambda} \mid+\frac{1}{8} \max \left\{\left|\log _{9} \frac{\rho_{A}\left(x_{\eta(i+1)}\right)}{\rho_{B}\left(x_{\eta(i+1)}\right)}\right|^{\lambda},\left|\log _{9} \frac{\sigma_{A}\left(x_{\eta(i+1)}\right)}{\sigma_{B}\left(x_{\eta(i+1)}\right)}\right|^{\lambda},\left|\log _{9} \frac{\tau_{A}\left(x_{\eta(i+1)}\right)}{\tau_{B}\left(x_{\eta(i+1)}\right)}\right|^{\lambda}\right\}\right]\right.}
\end{aligned}
$$

\subsubsection{Weighted distances between IMSs in the continuous case}

We find that all the above distance measures are based on discrete values. However, sometimes the universe of discourse and the weights of elements may be continuous. This subsection mainly discusses that case.

Let $A$ and $B$ be two IMSs in $X$ with the weight of $x \in X=[a, b]$ being $w(x)$, where $w(x) \in[0,1]$ and $\int_{b}^{a} w(x) d x=1$. We define a generalized continuous weighted distance, a continuous weighted Hamming distance 
and a continuous weighted Euclidean distance between the IMSs $A$ and $B$, respectively, as follows:

$$
\begin{gathered}
d_{g c w d}(A, B)=\left(\frac{1}{2^{\lambda+1}} \int_{a}^{b} w(x)\left(\left|\log _{9} \frac{\rho_{A}(x)}{\rho_{B}(x)}\right|^{\lambda}+\left|\log _{9} \frac{\sigma_{A}(x)}{\sigma_{B}(x)}\right|^{\lambda}+\left|\log _{9} \frac{\tau_{A}(x)}{\tau_{B}(x)}\right|^{\lambda}\right) d x\right)^{1 / \lambda}, \lambda>0 \\
d_{c w h d}(A, B)=\frac{1}{4} \int_{a}^{b} w(x)\left(\left|\log _{9} \frac{\rho_{A}(x)}{\rho_{B}(x)}\right|+\left|\log _{9} \frac{\sigma_{A}(x)}{\sigma_{B}(x)}\right|+\left|\log _{9} \frac{\tau_{A}(x)}{\tau_{B}(x)}\right|\right) d x \\
d_{\text {cwed }}(A, B)=\left(\frac{1}{8} \int_{a}^{b} w(x)\left(\left|\log _{9} \frac{\rho_{A}(x)}{\rho_{B}(x)}\right|^{2}+\left|\log _{9} \frac{\sigma_{A}(x)}{\sigma_{B}(x)}\right|^{2}+\left|\log _{9} \frac{\tau_{A}(x)}{\tau_{B}(x)}\right|^{2}\right) d x\right)^{1 / 2}
\end{gathered}
$$

If $w(x)=1 /(b-a)$ for any $x \in[a, b]$, then the above distances respectively reduce to a generalized continuous normalized weighted distance, a continuous normalized Hamming distance and a continuous normalized Euclidean distance, as follows:

$$
\begin{gathered}
d_{\text {gcnd }}(A, B)=\left(\frac{1}{2^{\lambda+1}(b-a)} \int_{a}^{b}\left(\left|\log _{9} \frac{\rho_{A}(x)}{\rho_{B}(x)}\right|^{\lambda}+\left|\log _{9} \frac{\sigma_{A}(x)}{\sigma_{B}(x)}\right|+\left|\log _{9} \frac{\tau_{A}(x)}{\tau_{B}(x)}\right|^{\lambda} d x\right)\right)^{1 / \lambda}, \lambda>0 \\
d_{\text {cnhd }}(A, B)=\frac{1}{4(b-a)} \int_{a}^{b}\left(\left|\log _{9} \frac{\rho_{A}(x)}{\rho_{B}(x)}\right|+\left|\log _{9} \frac{\sigma_{A}(x)}{\sigma_{B}(x)}\right|+\left|\log _{9} \frac{\tau_{A}(x)}{\tau_{B}(x)}\right|\right) d x \\
d_{\text {cned }}(A, B)=\left(\frac{1}{8(b-a)} \int_{a}^{b}\left(\left|\log _{9} \frac{\rho_{A}(x)}{\rho_{B}(x)}\right|^{2}+\left|\log _{9} \frac{\sigma_{A}(x)}{\sigma_{B}(x)}\right|^{2}+\left|\log _{9} \frac{\tau_{A}(x)}{\tau_{B}(x)}\right|^{2}\right) d x\right)^{1 / 2}
\end{gathered}
$$

On the basis of the Hausdorff metric, we can introduce a generalized continuous weighted Hausdorff distance, a continuous weighted Hamming-Hausdorff distance and a continuous weighted Euclidean-Hausdorff distance between $A$ and $B$ as follows:

$$
\begin{gathered}
d_{\text {gcwhaud }}(A, B)=\frac{1}{2}\left(\int_{a}^{b} w(x) \max \left\{\left|\log _{9} \frac{\rho_{A}(x)}{\rho_{B}(x)}\right|^{\lambda},\left|\log _{9} \frac{\sigma_{A}(x)}{\sigma_{B}(x)}\right|^{\lambda},\left|\log _{9} \frac{\tau_{A}(x)}{\tau_{B}(x)}\right|^{\lambda}\right\} d x\right)^{1 / \lambda}, \lambda>0 \\
d_{\text {cwhhaud }}(A, B)=\frac{1}{2} \int_{a}^{b} w(x) \max \left\{\left|\log _{9} \frac{\rho_{A}(x)}{\rho_{B}(x)}\right|,\left|\log _{9} \frac{\sigma_{A}(x)}{\sigma_{B}(x)}\right|,\left|\log _{9} \frac{\tau_{A}(x)}{\tau_{B}(x)}\right|\right\} d x
\end{gathered}
$$




$$
d_{\text {cwehaud }}(A, B)=\frac{1}{2}\left(\int_{a}^{b} w(x) \max \left\{\left|\log _{9} \frac{\rho_{A}(x)}{\rho_{B}(x)}\right|^{2},\left|\log _{9} \frac{\sigma_{A}(x)}{\sigma_{B}(x)}\right|^{2},\left|\log _{9} \frac{\tau_{A}(x)}{\tau_{B}(x)}\right|^{2}\right\} d x\right)^{1 / 2}
$$

If $w(x)=1 /(b-a)$ for any $x \in[a, b]$, then Eqs. (32)-(34) reduce respectively to a generalized continuous normalized Hausdorff distance, a continuous normalized Hamming-Hausdorff distance and a continuous normalized Euclidean-Hausdorff distance between $A$ and $B$ as follows:

$$
\begin{gathered}
d_{\text {gcnwhaud }}(A, B)=\frac{1}{2}\left(\frac{1}{b-a} \int_{a}^{b} \max \left\{\left|\log _{9} \frac{\rho_{A}(x)}{\rho_{B}(x)}\right|^{\lambda},\left|\log _{9} \frac{\sigma_{A}(x)}{\sigma_{B}(x)}\right|^{\lambda},\left|\log _{9} \frac{\tau_{A}(x)}{\tau_{B}(x)}\right|^{\lambda}\right\} d x\right)^{1 / \lambda}, \lambda>0 \\
d_{\text {cnhhaud }}(A, B)=\frac{1}{2(b-a)} \int_{a}^{b} \max \left\{\left|\log _{9} \frac{\rho_{A}(x)}{\rho_{B}(x)}\right|,\left|\log _{9} \frac{\sigma_{A}(x)}{\sigma_{B}(x)}\right|,\left|\log _{9} \frac{\tau_{A}(x)}{\tau_{B}(x)}\right|\right\} d x \\
d_{\text {crehaud }}(A, B)=\frac{1}{2}\left(\frac{1}{b-a} \int_{a}^{b} \max \left\{\left|\log _{9} \frac{\rho_{A}(x)}{\rho_{B}(x)}\right|^{2},\left|\log _{9} \frac{\sigma_{A}(x)}{\sigma_{B}(x)}\right|^{2},\left|\log _{9} \frac{\tau_{A}(x)}{\tau_{B}(x)}\right|^{2}\right\} d x\right)^{1 / 2}
\end{gathered}
$$

We can, of course, derive some hybrid continuous weighted distance measures, such as a generalized hybrid continuous weighted distance, a hybrid continuous weighted Hamming distance and a hybrid continuous weighted Euclidean distance between $A$ and $B$ as follows:

$$
\begin{aligned}
& d_{\text {ghcovd }}(A, B)=\left(\int_{a}^{b} w(x)\left(\frac{1}{2^{\lambda+2}}\left(\left|\log _{9} \frac{\rho_{A}\left(x_{i}\right)}{\rho_{B}\left(x_{i}\right)}\right|^{\lambda}+\left|\log _{9} \frac{\sigma_{A}\left(x_{i}\right)}{\sigma_{B}\left(x_{i}\right)}\right|^{\lambda}+\left|\log _{9} \frac{\tau_{A}\left(x_{i}\right)}{\tau_{B}\left(x_{i}\right)}\right|^{\lambda}\right)+\frac{1}{2^{\lambda+1}} \max \left\{\left|\log _{9} \frac{\rho_{A}\left(x_{i}\right)}{\rho_{B}\left(x_{i}\right)}\right|^{\lambda},\left|\log _{9} \frac{\sigma_{A}\left(x_{i}\right)}{\sigma_{B}\left(x_{i}\right)}\right|^{\lambda},\left|\log _{9} \frac{\tau_{A}\left(x_{i}\right)}{\tau_{B}\left(x_{i}\right)}\right|\right\}\right) d x\right)^{\lambda}, \lambda>0 \\
& \left.d_{\text {hcwhd }}(A, B)=\int_{a}^{b} w(x)\left(\frac{1}{8}\left(\left|\log _{9} \frac{\rho_{A}\left(x_{i}\right)}{\rho_{B}\left(x_{i}\right)}\right|+\left|\log _{9} \frac{\sigma_{A}\left(x_{i}\right)}{\sigma_{B}\left(x_{i}\right)}\right|+\left|\log _{9} \frac{\tau_{A}\left(x_{i}\right)}{\tau_{B}\left(x_{i}\right)}\right|\right)+\frac{1}{4} \max \left\{\left|\log _{9} \frac{\rho_{A}\left(x_{i}\right)}{\rho_{B}\left(x_{i}\right)}\right|,\left|\log _{9} \frac{\sigma_{A}\left(x_{i}\right)}{\sigma_{B}\left(x_{i}\right)}\right|, \mid \log _{9} \frac{\tau_{A}\left(x_{i}\right)}{\tau_{B}\left(x_{i}\right)}\right)\right\}\right\} d x \\
& d_{\text {hcwed }}(A, B)=\left(\int_{a}^{b} w(x)\left(\frac{1}{16}\left(\left|\log _{9} \frac{\rho_{A}\left(x_{i}\right)}{\rho_{B}\left(x_{i}\right)}\right|^{2}+\left|\log _{9} \frac{\sigma_{A}\left(x_{i}\right)}{\sigma_{B}\left(x_{i}\right)}\right|^{2}+\left|\log _{9} \frac{\tau_{A}\left(x_{i}\right)}{\tau_{B}\left(x_{i}\right)}\right|^{2}\right)+\frac{1}{8} \max \left\{\left|\log _{9} \frac{\rho_{A}\left(x_{i}\right)}{\rho_{B}\left(x_{i}\right)}\right|^{2}\left|\log _{9} \frac{\sigma_{A}\left(x_{i}\right)}{\sigma_{B}\left(x_{i}\right)}\right|^{2},\left|\log _{9} \frac{\tau_{A}\left(x_{i}\right)}{\tau_{B}\left(x_{i}\right)}\right|^{2}\right\}\right) d x\right\}^{1 / 2}
\end{aligned}
$$

If $w(x)=1 /(b-a)$ for any $x \in[a, b]$, then Eqs. (38)-(40) reduce respectively to a generalized hybrid continuous normalized distance, a hybrid continuous normalized Hamming distance and a hybrid continuous normalized Euclidean distance between $A$ and $B$ as follows:

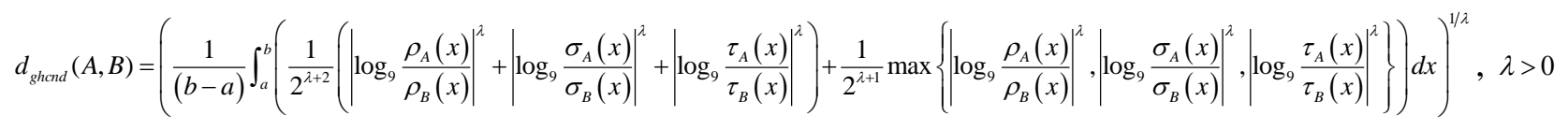




$$
\begin{gathered}
\left.d_{\text {hcwh }}(A, B)=\frac{1}{(b-a)} \int_{a}^{b}\left(\frac{1}{8}\left(\left|\log _{9} \frac{\rho_{A}(x)}{\rho_{B}(x)}\right|+\left|\log _{9} \frac{\sigma_{A}(x)}{\sigma_{B}(x)}\right|+\left|\log _{9} \frac{\tau_{A}(x)}{\tau_{B}(x)}\right|\right)+\frac{1}{4} \max \left\{\mid \log _{9} \frac{\rho_{A}(x)}{\rho_{B}(x)}\right),\left|\log _{9} \frac{\sigma_{A}(x)}{\sigma_{B}(x)}\right|,\left|\log _{9} \frac{\tau_{A}(x)}{\tau_{B}(x)}\right|\right\}\right) d x \\
d_{\text {hcwed }}(A, B)=\left(\frac{1}{(b-a)} \int_{a}^{b}\left(\frac{1}{16}\left(\left|\log _{9} \frac{\rho_{A}(x)}{\rho_{B}(x)}\right|^{2}+\left|\log _{9} \frac{\sigma_{A}(x)}{\sigma_{B}(x)}\right|^{2}+\left|\log _{9} \frac{\tau_{A}(x)}{\tau_{B}(x)}\right|^{2} \mid+\frac{1}{8} \max \left\{\left|\log _{9} \frac{\rho_{A}(x)}{\rho_{B}(x)}\right|^{2},\left|\log _{9} \frac{\sigma_{A}(x)}{\sigma_{B}(x)}\right|^{2},\left|\log _{9} \frac{\tau_{A}(x)}{\tau_{B}(x)}\right|^{2}\right\}\right) d x\right)^{1 / 2}\right.
\end{gathered}
$$

Note. In Section 3.2, various weighted distance measures for IMSs based on the generalized weighted Hausdorff distance and the generalized hybrid weighted distance were investigated. It is easy to demonstrate that all the proposed weighted distance measures also have the properties shown in Properties 1 and 2.

Example 1. Let $A$ and $B$ be two IMSs in $X=\left\{x_{1}, x_{2}\right\}$ and $A=\{(1 / 6,4),(2,1 / 6)\}, B=\{(6,1 / 9),(7,1 / 9)\}$. Their weight vector is $w=(1 / 3,2 / 3)^{T}$.

(1) Using Eq. (11), we can obtain the generalized weighted Hausdorff distance between $A$ and $B$. The results are shown in Fig. 1. The left side of Fig. 1 shows the variations and distributions of the generalized weighted Hausdorff distance between $A$ and $B$ when $0 \leq \lambda \leq 1000$ whilst the right side shows these data for $0 \leq \lambda \leq 100$. When $\lambda=1$ and $\lambda=2$, we can obtain the weighted Hamming-Hausdorff distance and the weighted EuclideanHausdorff distance between $A$ and $B: d_{\text {whhaud }}(A, B)=0.4619, d_{\text {wehaud }}(A, B)=0.5252$.
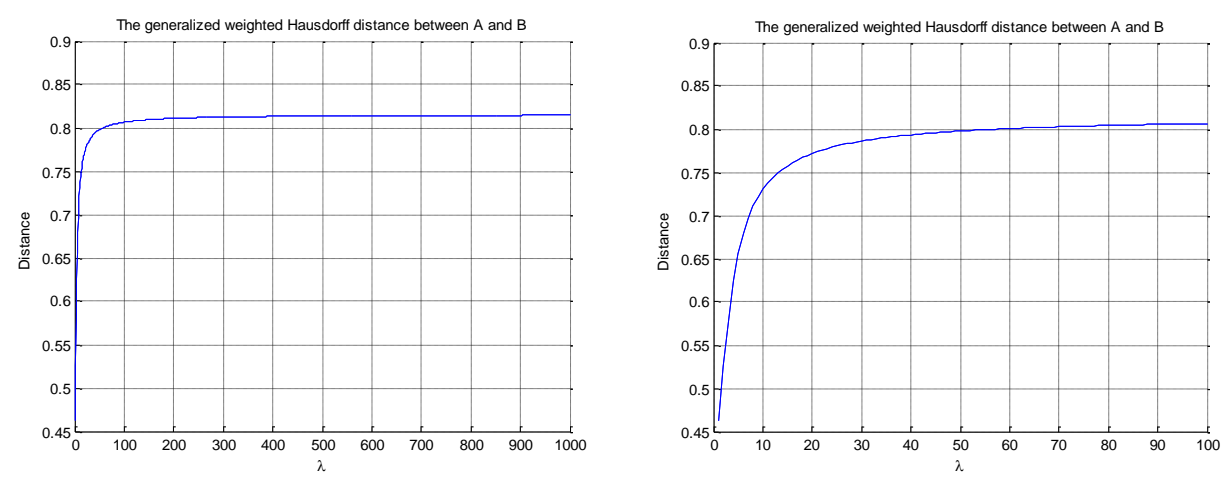

Fig. 1 The generalized weighted Hausdorff distance between $A$ and $B$

(2) Using Eq. (16), we can obtain the generalized hybrid weighted distance between $A$ and $B$. The results are shown in Fig. 2. The left side of Fig. 2 shows the variations and distributions of the generalized hybrid weighted distance between $A$ and $B$ when $0 \leq \lambda \leq 1000$ whilst the right side shows the data for $0 \leq \lambda \leq 100$. We can obtain the hybrid weighted Hamming distance and the hybrid weighted Euclidean distance between $A$ and $B$ for $\lambda=1$ and $\lambda=2$, respectively: $d_{h w h d}(A, B)=0.4619$ and $d_{h w e d}(A, B)=0.5195$. 

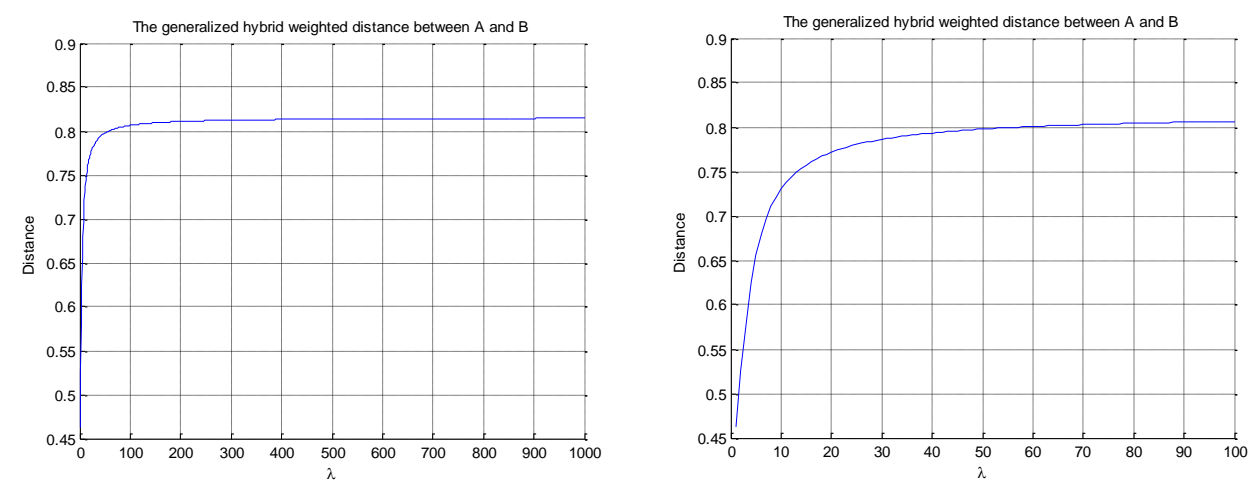

Fig. 2 The generalized hybrid weighted distance between $A$ and $B$

We can see from Fig. 1 and Fig. 2 that the variations and distributions of these generalized measures of the distance between $A$ and $B$ are almost the same. Both Figs. 1 and 2 exhibit a slow decrease in the rate of growth of the distance between $A$ and $B$, whereas all the distances become gradually larger as the value of $\lambda$ increases, and they ultimately converge at approximately 0.82 . When $\lambda=1$ and $\lambda=100$, we obtain the same distance values, i.e., 0.4619 and 0.82 , representing the minimum and maximum values, respectively. It should be noted that both the generalized weighted Hausdorff distance and the weighted Minkowski distance make up the generalized hybrid weighted distance. Calculation of the generalized hybrid weighted distance is more complex and hence more timeconsuming than calculation of the generalized weighted Hausdorff distance. Hybrid distance measures are more robust than single distances. We can choose which distance measure to use based on the demands of a particular DM.

Example 2. Let $A=\{(5,1 / 7),(6,1 / 7),(1 / 5,4)\}, B=\{(2,1 / 7),(1 / 7,5),(1 / 6,5)\}$ and $C=\{(6,1 / 6),(4,1 / 8),(1 / 7,2)\}$ be three IMSs, and $w_{i}=(1 / 3,1 / 6,1 / 2)^{T}$ be their weight vector. Four distance measures - the Minkowski distance, the weighted Minkowski distance, the generalized hybrid distance, and the generalized hybrid weighted distance - are used to determine the values of $d(A, B), d(A, C)$, and $d(B, C)$, and the results are shown in Figs. $3-5$, respectively. 

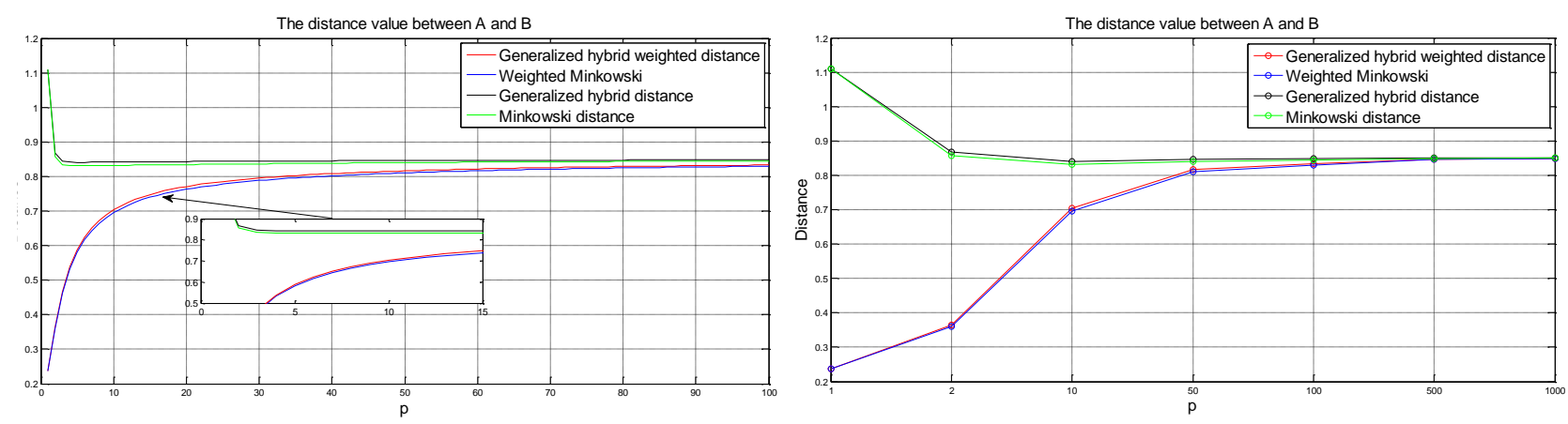

Fig. 3 The distance between $A$ and $B$
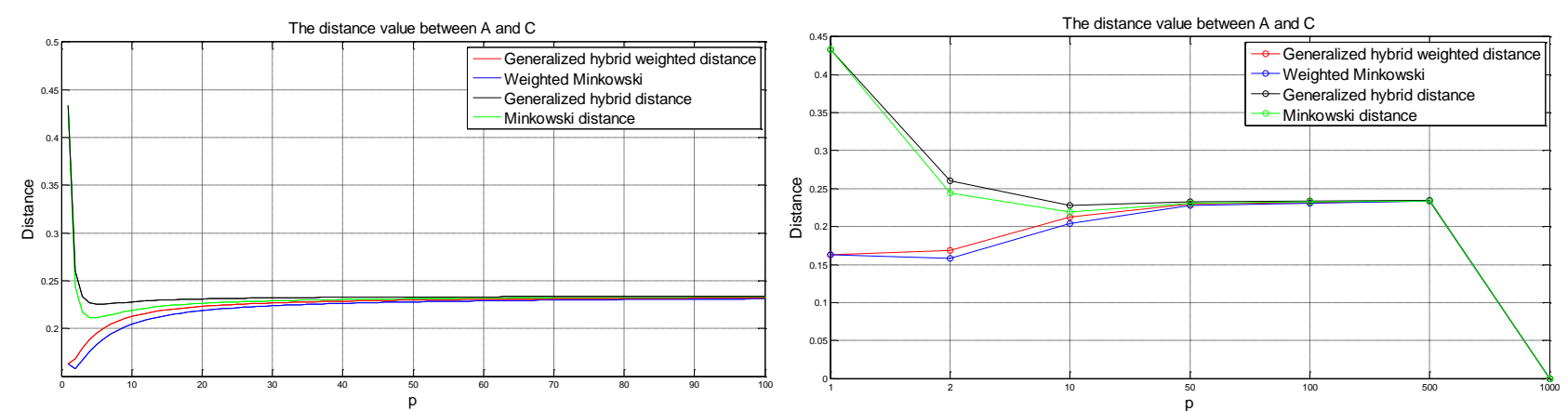

Fig. 4 The distance between $A$ and $C$
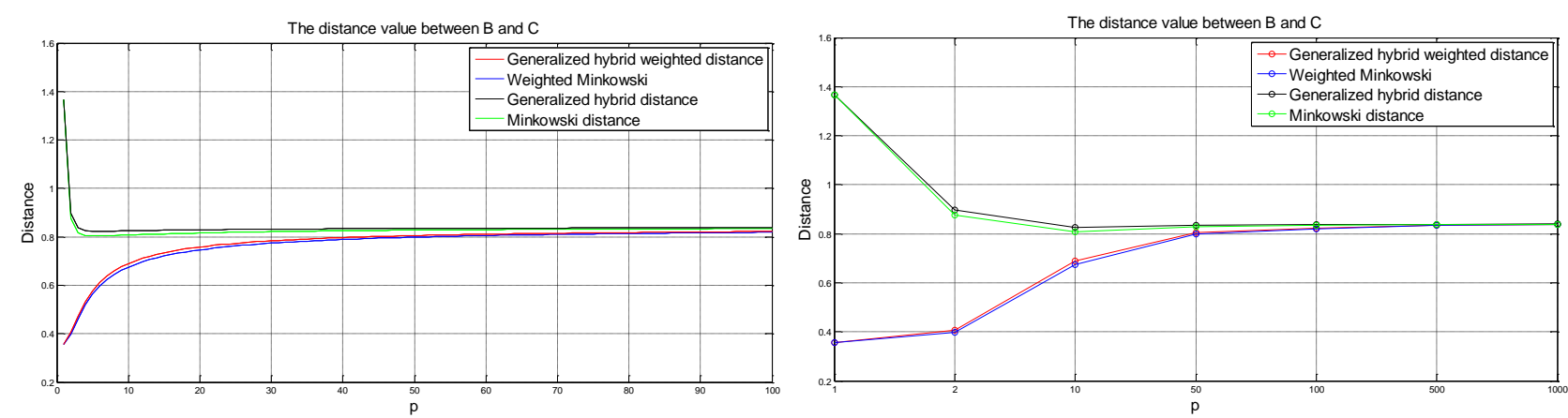

Fig. 5 The distance between $B$ and $C$

The left side of Fig. 3 shows the distance between $A$ and $B$ when $0 \leq \lambda \leq 100$ whilst the right side shows the corresponding distance with $\lambda=1,2,10,50,100,500,1000$. Corresponding values for the distances between $A$ and $C$ and $B$ and $C$ are shown in Figs. 4 and 5 respectively. Based on these figures we can draw the following conclusions:

(1) Using different distance measures we obtain different values for the distance between IMSs. For instance, the generalized hybrid distance and the weighted Minkowski distance provide the biggest and smallest values respectively.

(2) The values of weighted distance measures gradually increase with $\lambda$, whereas values of unweighted distance measures decrease. Note also that the values of the weighted distances are smaller than those of the unweighted distances. 
(3) The right sides of Figs. 3-5 show that the differences between the values of the different distance measures gradually decrease as the values of $\lambda$ increase and finally tend to zero. Meanwhile, when $\lambda=1$ and $\lambda=2$, we have $d(B, C)>d(A, B)>d(A, C)$; whilst for $\lambda=10, \lambda=50, \lambda=100, \lambda=500, \lambda=1000$, we have $d(A, B)>d(B, C)>d(A, C)$. This means that the proposed distance measures, i.e., the generalized hybrid distance and the generalized hybrid weighted distance, provide effective measurements of the difference between IMSs.

Example 3. (Continued from Example 2) Assume that $w=(1 / 6,1 / 3,1 / 2)^{T}$ is the weight vector of the ordered positions of the distances $\Delta\left(A\left(x_{i}\right), B\left(x_{i}\right)\right)$, where

$$
\Delta\left(A\left(x_{i}\right), B\left(x_{i}\right)\right)=\left(\left|\log _{9} \frac{\rho_{A}\left(x_{\eta(i-1)}\right)}{\rho_{B}\left(x_{\eta(i-1)}\right)}\right|^{\lambda}+\left|\log _{9} \frac{\sigma_{A}\left(x_{\eta(i-1)}\right)}{\sigma_{B}\left(x_{\eta(i-1)}\right)}\right|^{\lambda}+\left|\log _{9} \frac{\tau_{A}\left(x_{\eta(i-1)}\right)}{\tau_{B}\left(x_{\eta(i-1)}\right)}\right|^{\lambda}\right) \text {, for } i=1,2,3
$$

(I) Using Eq. (17), we can obtain the generalized ordered weighted distance between $A$ and $B$. When $\lambda=1$ and $\lambda=2$, we have $d_{\text {owhd }}(A, B)=0.2366$ and $d_{\text {owed }}(A, B)=0.3613$.

(II) Using Eq. (20), we can obtain the generalized ordered weighted Hausdorff distance between $A$ and $B$. When $\lambda=1$ and $\lambda=2$, we have $d_{\text {owhhaud }}(A, B)=0.2367$ and $d_{\text {owehaud }}(A, B)=0.3693$.

(III) Using Eq. (23), we can obtain the hybrid generalized ordered weighted distance between $A$ and $B$. When $\lambda=1$ and $\lambda=2$, we have $d_{\text {howhd }}(A, B)=0.2367$ and $d_{\text {howed }}(A, B)=0.3653$.

Examples 2 and 3 show that the values of the weighed distance measures, i.e., those given by Eqs. (17), (20) and (23), are smaller than those of the unweighted distance measures, i.e., Eqs. (2) and (10). As mentioned above, a prominent characteristic of ordered weighted distance measures is that they can modify the influence of unduly large or small deviations on the aggregation results by assigning them low or high weights (Xu \& Chen, 2008). In such cases, the obtained results are closer to DMs' preferences and more consistent with the real situation. In addition, this section further investigates the use of continuous weighted distance with IMSs, which provides a solution to the problem of the continuity of weight vector for attributes. The strength, characteristics and applicability of these proposed distance measures are further summarized in Table 1. On this basis, the choice of distance measure can be based on the demands of the DMs.

Table 1 Comparisons of the proposed IM distance measures

\begin{tabular}{|c|c|c|c|c|}
\hline Distance measures & & Strength & Characteristics & Applicability \\
\hline Unweighted form & Generalized Hausdorff distance & Moderate & Regardless of weight & MCDM problems with \\
\hline
\end{tabular}


equal weights of the

DMs or criteria;

Regardless of weight

It can be widely used to

Generalized weighted Hausdorff Both the weight vectors

distance Strong

of DMs and criteria are

measure the weighted

distance between considered

IMSs/IMNs

It can diminish or

enhance the influence of The case considering

unduly large or small

deviations

the orders of criteria

Weighted form

Ordered weighted Hausdorff Moderate
distance

aggregation results

It is mainly used to tackle the case that the

Continuous weighted Hausdorff distance

Weak

Continuous values universe of discourse and the weights of elements are continuous

Note that the term "strength" in Table 1 denotes the strength of practicality, that is, the frequency of application in real case. Moreover, the hybrid distance measures for IMSs/IMNs have stronger robustness compared with single forms such as the Hausdorff distance, Hamming distance, and Euclidean distance.

\section{Two distance-based methods for MCDM problems with intuitionistic multiplicative information}

A MCDM can be described as follows: let $X=\left\{X_{1}, X_{2}, \cdots, X_{m}\right\}$ be a discrete set of options, where the DMs need to choose the best option from $X$ according to the criterion set $C=\left\{C_{1}, C_{2}, \cdots, C_{n}\right\}$. Suppose that the values for the criteria, given by the DMs, are represented by the IMNs $\left\{\left(\rho_{X_{i}}\left(C_{j}\right), \sigma_{X_{i}}\left(C_{j}\right)\right) \mid i=1,2, \cdots, m, j=1,2, \cdots, n\right\}$, where $\rho_{X_{i}}\left(C_{j}\right)$ and $\sigma_{X_{i}}\left(C_{j}\right)$ are the degrees of membership and non-membership, respectively, of option $X_{i}$ based on criterion $C_{j}$, and satisfy: $1 / 9 \leq \rho_{X_{i}}\left(C_{j}\right), \sigma_{X_{i}}\left(C_{j}\right) \leq 9,0<\rho_{X_{i}}\left(C_{j}\right) \sigma_{X_{i}}\left(C_{j}\right) \leq 1$. Together these IMNs constitute an intuitionistic multiplicative decision matrix (IMDM) $R=\left(r_{i j}\right)_{m \times n}=\left(\rho_{X_{i}}\left(C_{j}\right), \sigma_{X_{i}}\left(C_{j}\right)\right)_{m \times n}$. In this section, based on the proposed measures of the distance between IMSs, we propose two methods of solving MCDM problems with intuitionistic multiplicative information.

\subsection{The distance-based IM-TOPSIS method}

TOPSIS (Chen \& Hwang, 1992) is a well-known method and is widely used in solving MCDM problems. With this method, the chosen option should be furthest from the negative-ideal solution and closest to the positive- 
ideal solution. In other words, the distance measure plays an important role in this method. There has been a lot of work done on the TOPSIS method and it can be divided into two categories: (i) extensions of the TOPSIS method to multiple fuzzy sets (Liao \& Xu, 2015), including IFSs, interval-valued IFSs, Pythagorean fuzzy sets, HFSs and HFLTSs; (ii) applications in a wide variety of fields, such as the healthcare industry, manufacturing, business investment, military training, financial management of government and national policies.

By combining the extant research results with the proposed measures of distance between IMSs we can develop a distance-based, intuitionistic multiplicative TOPSIS (IM-TOPSIS) method. The general procedures of the IM-TOPSIS are shown below.

\section{Algorithm 1}

Step 1. Build the IMDM based on the initial intuitionistic multiplicative evaluation values given by the DM.

Step 2. Determine the intuitionistic multiplicative positive-ideal solution (IMPIS) and the intuitionistic multiplicative negative ideal solution (IMNIS). Note that the evaluation criteria can be classified into benefit criteria and cost criteria. We need to do some unification before carrying out further calculations. The values of the cost criterion $C_{j}$ can be translated to their opposites as:

$$
\left(\rho_{X_{i}}^{\prime}\left(C_{j}\right), \sigma_{X_{i}}^{\prime}\left(C_{j}\right)\right)=\left(\sigma_{X_{i}}\left(C_{j}\right), \rho_{X_{i}}\left(C_{j}\right)\right)
$$

with $C_{j}$ being the cost criterion.

In this way we can obtain a unified IMDM $R^{\prime}$. To facilitate the presentation, we will assume that all the criteria are benefit criteria, if not they could be translated as illustrated above. In this case, the IMPIS $X^{+}$and the IMNIS $X^{-}$can be defined as:

$$
X^{+}=\left[C_{1}^{+}, C_{2}^{+}, \cdots, C_{j}^{+}, \cdots C_{n}^{+}\right], X^{-}=\left[C_{1}^{-}, C_{2}^{-}, \cdots, C_{j}^{-}, \cdots C_{n}^{-}\right]
$$

where $C_{j}^{+}=\max _{i} C_{i j}=\left(\rho_{j}^{+}, \sigma_{j}^{+}\right), C_{j}^{-}=\min _{i} C_{i j}=\left(\rho_{j}^{-}, \sigma_{j}^{-}\right)(j=1,2, \cdots, n)$. Given that the criteria are sometimes conflicting; it is realistic to assume that such an IMPIS does not exist; if it did the decision would be trivial.

Step 3. Calculate the distance between each option and the IMPIS, $d\left(X_{i}, X^{+}\right)$, and the distance between each option and the IMNIS, $d\left(X_{i}, X^{-}\right)$, using the distance measures introduced in Section 3.

Step 4. Calculate the relative closeness coefficients $c\left(X_{i}\right)(i=1,2, \cdots, m)$ for all options, where 


$$
c\left(X_{i}\right)=d\left(X_{i}, X^{-}\right) /\left(d\left(X_{i}, X^{+}\right)+d\left(X_{i}, X^{-}\right)\right)
$$

Step 5. Sort $c\left(X_{i}\right)(i=1,2, \cdots, m)$ in descending order. A large value for the relative closeness coefficient $c\left(X_{i}\right)$ implies that the option $X_{i}$ is close to the IMPIS and relatively distant from the IMNIS. Thus all options can be ranked in descending order of their relative closeness coefficient values. The optimal option is the one with the highest closeness coefficient value.

\subsection{The distance-based IM-VIKOR method}

The VIKOR (Vlsekriterijumska Optimizacija I Kompromisno Resenje in Serbian, meaning multi-criteria optimization and compromise solution) method, which yields compromise solution(s) based on the ideal solution, was first proposed by Opricovic (1998). Mardani et al. (2016) gave a comprehensive overview of the VIKOR method based on the papers published from 2004 to 2015. Our review of the literature indicated that the VIKOR method has been applied to MCDM problems in many different areas, including material selection, supplier selection, renewable energy plan selection and information security risk control assessment. In addition, the VIKOR method has been extended to other information representation environments, such as IFSs, HFSs, HFLTSs and interval neutrosophic sets (Liao \& Xu, 2015; Liao \& Xu, 2013; Liao, Xu \& Zeng, 2015; Bausys \& Zavadskas, 2015).

However, there has been no research on the VIKOR method with IMSs. Hence we have developed a distancebased intuitionistic multiplicative VIKOR (IM-VIKOR) method, by combining the existing research results with the proposed measures of distance between IMSs. This method involves the following steps:

\section{Algorithm 2}

Steps 1-2. As for Algorithm 1.

Step 3. Calculate the values of the intuitionistic multiplicative group utility measure $I M G U_{i}$ and the intuitionistic multiplicative individual regret measure $I M I R_{i}$ for all options, defined in terms of the measures of distance between IMSs introduced in Section 3, and shown below (here we choose the Euclidean distance as an example): 


$$
\begin{gathered}
I M G U_{i}=\sum_{j=1}^{n} w_{j} \frac{C_{j}^{+}-r_{i j}}{C_{j}^{+}-C_{j}^{-}}=\sum_{j=1}^{n} w_{j}\left(\sqrt{\frac{1}{8}\left(\left|\log _{9} \frac{\rho_{j}^{+}}{\rho_{j}^{i}}\right|^{2}+\left|\log _{9} \frac{\sigma_{j}^{+}}{\sigma_{j}^{i}}\right|^{2}+\left|\log _{9} \frac{\tau_{j}^{+}}{\tau_{j}^{i}}\right|^{2}\right)} / \sqrt{\frac{1}{8}\left(\left|\log _{9} \frac{\rho_{j}^{+}}{\rho_{j}^{-}}\right|^{2}+\left|\log _{9} \frac{\sigma_{j}^{+}}{\sigma_{j}^{-}}\right|^{2}+\left|\log _{9} \frac{\tau_{j}^{+}}{\tau_{j}^{-}}\right|^{2}\right)}\right) \\
\left.\operatorname{IMIR}_{i}=\max _{j}\left\{w_{j} \frac{C_{j}^{+}-r_{i j}}{C_{j}^{+}-C_{j}^{-}}\right\}=\max _{j}\left\{w_{j}\left(\sqrt{\frac{1}{8}\left(\left|\log _{9} \frac{\rho_{j}^{+}}{\rho_{j}^{i}}\right|^{2}+\left|\log _{9} \frac{\sigma_{j}^{+}}{\sigma_{j}^{i}}\right|^{2}+\left|\log _{9} \frac{\tau_{j}^{+}}{\tau_{j}^{i}}\right|^{2}\right.}\right) / \sqrt{\frac{1}{8}\left(\left|\log _{9} \frac{\rho_{j}^{+}}{\rho_{j}^{-}}\right|^{2}+\left|\log _{9} \frac{\sigma_{j}^{+}}{\sigma_{j}^{-}}\right|^{2}+\left|\log _{9} \frac{\tau_{j}^{+}}{\tau_{j}^{-}}\right|^{2}\right)}\right)\right\}
\end{gathered}
$$

Desirable options should have values of these two measures that are as small as possible. Minimization of $I M G U_{i}$ implies maximization of the group intuitionistic multiplicative group utility, whereas to minimize $I M I R_{i}$ is to minimize individual regret.

Step 4. Calculate the value of the intuitionistic multiplicative compromise measure $I M C_{i}$ of option $X_{i}$ :

$$
I M C_{i}=v \frac{I M G U_{i}-I M G U^{-}}{I M G U^{+}-I M G U^{-}}+(1-v) \frac{I M I R_{i}-I M I R^{-}}{I M I R^{+}-I M I R^{-}}
$$

where $I M G U^{-}=\min _{i} I M G U_{i}, I M G U^{+}=\max _{i} I M G U_{i}, I M I R^{-}=\min _{i} I M I R_{i}, I M I R^{+}=\max _{i} I M I R_{i}$. The coefficient $v \in[0,1]$ denotes the trade-off the DM makes between group utility and individual regret over the conflicting criteria: if $0.5<v<1$, then the DM prefers to maximize the group utility; if $0<v<0.5$, then the DM pays more attention to minimization of individual regret; if $v=0.5$, then the DM give equal weight to group utility and individual regret. The smaller the value of $I M C_{i}$ is, the better the option should be.

Step 5. Rank the options in decreasing order of the values of $I M G U_{i}, I M I R_{i}$ and $I M C_{i}$, producing three ranking lists.

Step 6. Propose compromise solution(s) for the MCDM problem.

(1) There is only one compromise solution $X^{(1)}$, which is the first when it is ranked in ascending order of the values of $I M C_{i}$, and satisfying the following two conditions:

C1: $\operatorname{IMC}\left(X^{(2)}\right)-\operatorname{IMC}\left(X^{(1)}\right) \geq \Delta$, where $X^{(2)}$ is the second-ranked option in the list of values of the intuitionistic multiplicative compromise measure $I M C_{i} ; \Delta=1 /(m-1)$ is the threshold, showing the acceptable advantage of the compromise option $X^{(1)}$, with $m$ being the number of options. If $m \leq 4$, then we let $\Delta=0.25$ in order to identify the unique compromise solution (Opricovic \& Tzeng, 2004).

C2: The option $X^{(1)}$ is also the best in terms of the ranking of the intuitionistic multiplicative group utility 
measure $I M G U_{i}$ and the intuitionistic multiplicative individual regret measure $I M I R_{i}$. This condition is used to guarantee that the unique compromise solution is stable.

(2) There is more than one compromise solution if the above two conditions are not fully satisfied. If the first condition does not hold, then $X^{(1)}, X^{(2)}, \cdots, X^{(M)}$ are compromise solutions where $X^{(M)}$ meets the condition $I M C\left(X^{(M)}\right)-I M C\left(X^{(1)}\right)<\Delta$. In this case, all the options $X^{(1)}, X^{(2)}, \cdots, X^{(M)}$ are very close to each other in terms of the intuitionistic multiplicative compromise measure $I M C_{i}$. If only the second condition does not hold, then options $X^{(1)}$ and $X^{(2)}$ are the compromise solutions.

The flow charts of the distance-based IM-TOPSIS method and IM-VIKOR method are shown in Fig. 6 (a)-(b), respectively.
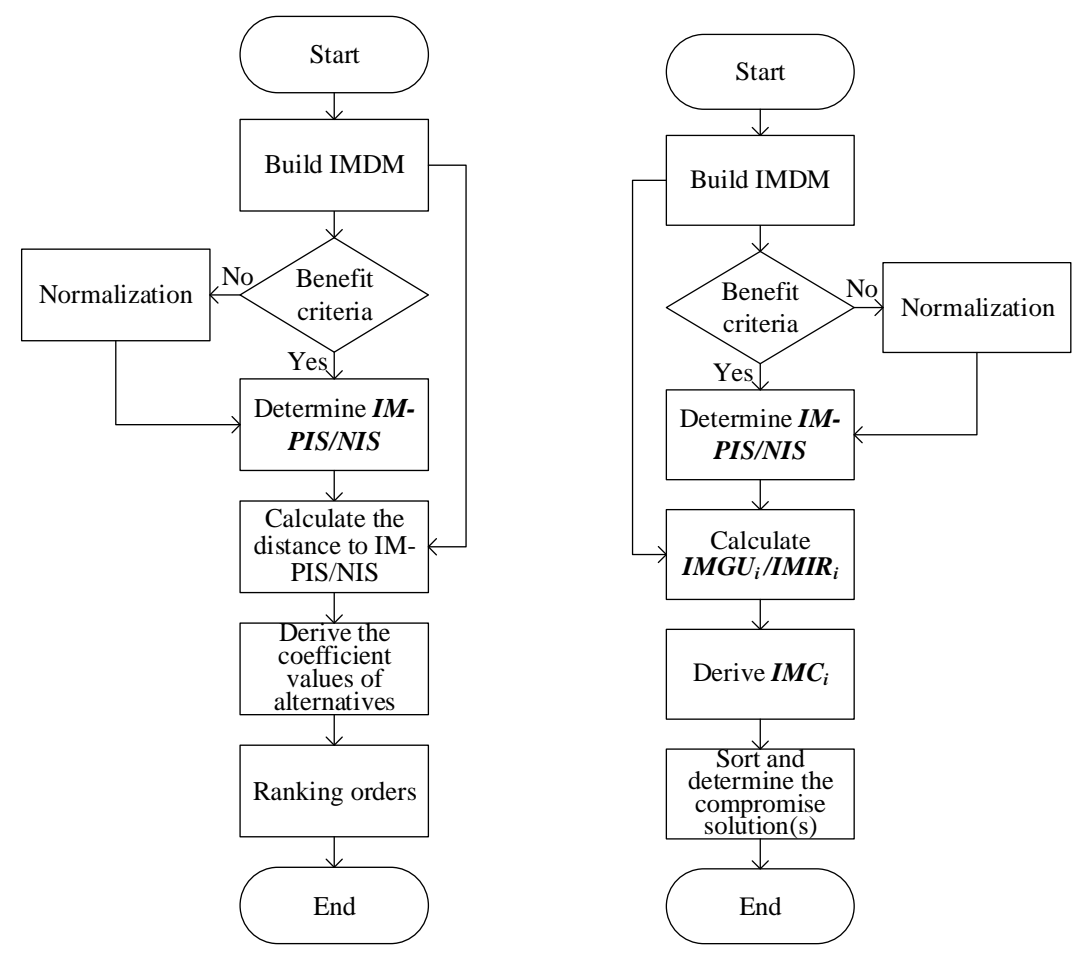

(a) Distance-based IM-TOPSIS method

(b) Distance-based IM-VIKOR method

Fig. 6 Flow charts of the intuitionistic multiplicative TOPSIS and VIKOR methods

In this section, we extend the TOPSIS method and VIKOR method into the IMS environment. The new parts between the proposed algorithms and their traditional forms can be respectively justified as follows: (1) With the new method, DMs can express their evaluations in IMSs rather than real numbers or other forms. In this sense, it has a stronger ability to depict MCDM problems with unbalanced or asymmetric situations; (2) To measure the 
distances in the TOPSIS method or VIKOR method (Step 3 in both of them), all the proposed distance measures for IMSs can be used. In this regard, DMs can choose an appropriate distance measure of IMSs according to their actual demand in real case.

\section{A case study: Healthcare management at WCH}

In this section, a case study concerning hospital management is used to illustrate the application of the proposed MCDM methods as well as the distance measures between IMSs.

\subsection{Case introduction}

$\mathrm{WCH}$, located in the southwest of China, is not only the largest single-site hospital in the world but also a leading medical center that treats various complicated and severe cases especially in the fields of living donor liver transplantation, severe acute pancreatitis and clinical anesthesia. It is a comprehensive hospital classed as Grade-A Class-Three in China. It was the first hospital in China to be accredited by the American Society of Pathologists (ASP). The WCH is also seeking to become a national center for medical scientific research and technology innovation. The WCH takes a large number of patients from Sichuan province and the surrounding provinces and cities due to its advanced medical technology, equipment and wealth of medical resources. According to the hospital's own statistics there are about 18,000 outpatients and more than 6000 patients waiting for a bed on any given day.

In recent years the continuing development of the $\mathrm{WCH}$ has led to the emergence of some management problems. We know that beds are an extremely scarce hospital resource and that the main goal of hospital bed management is to maximize utilization of resources. Every year the WCH admits nearly 200,000 inpatients, but at least thirty percent of this number (about 60,000 inpatients) do not need to be treated in the WCH. A small proportion of the inpatients suffers from severe disease or special value of clinical pathology, but most of them suffer from less serious conditions, such as toothache and minor trauma. These patients are taking up high-quality medical resources, which means that there are no beds for other patients needing hospital treatment; on the other hand treating patients with simple diseases (appendicitis, gallbladder problems etc.) allows the WCH to participate in development of medical technology and in scientific research. The conflict between the large number of patients seeking to benefit from the excellent resources of the $\mathrm{WCH}$ despite having no clinical need for them and the 
scarcity of medical resource has become a more and more serious problem for the $\mathrm{WCH}$.

The WCH admission center was established in 2011 to deal with this tricky problem. The center has two main objectives: (i) to optimize the hospitalization admission process and select patients; (ii) to improve the efficiency with which resources are used and ensure the fair and intelligent use of medical resources. The first stage of the inpatient admission process is that the patient is diagnosed in an outpatient department, and then registered by the admission center to reserve waiting outside the hospital. The system is designed to prioritize admission of patients who have the greatest need of a bed or the most serious clinical need. The triage of patients should resolve the conflict between the supply of hospital resources and the considerable demand of patients. Every day there are many patients in admission center. Ranking the patients is an onerous task, based on highly subjective criteria. We therefore need an efficient, scientifically based decision support technique to support the works and decide which patients should be prioritized, which patients should wait for admission, and which patients should not be admitted.

A review of the relevant literature showed that many scholars in other countries have considered this issue. Solans-Domènech et al. (2013) developed a priority scoring system for patients waiting for elective surgery, based on the condition of the patient and other, situational factors. Rahimi et al. (2015) proposed a risk-based framework for patient prioritization that involved using soft fuzzy sets to help hospitals to select high-risk patients. This not only ensures that the risks associated with long waiting times and delayed treatment are borne by the patients least likely to be adversely affected, but also increases the overall quality of medical service. Rahimi et al. (2016) presented a new approach to decisions where the information and risks are uncertain, which takes into account the opinions of surgery team members and patients. Ashour and Kremer (2016) developed a dynamic grouping and prioritization algorithm based on existing triage algorithms, which can not only reduce emergency room waiting times and the average length of stay but also reduces the observed risks of delayed treatment. It can satisfy the demands of emergency departments. There is, however, a significant difference between the medical systems for which these tools were designed and the Chinese medical system, which means that many frameworks or prioritization approaches are not suitable for use in the Chinese healthcare context. Hence, we further view the relevant literatures regarding China's hospital management. Zhang et al. (2016) used a hesitant fuzzy linguistic VIKOR method to solve the inpatient admission assessment problem at the $\mathrm{WCH}$, based on the following criteria: "urgency", "need for hospitalization", "value of clinical pathology". However, these authors did not consider other risks, limitations on the patient's activity or time spent on the waiting list. After consulting experts and staff in the 
admission center and referring to the previous literatures, we developed an approach that would address these shortcomings, specifying the following admission criteria and corresponding weights.

- $C_{1}$ (Value of Clinical Pathology, $w_{1}=0.15$ ). Dealing with patients who suffer from difficult or complicated disease can promote the development of iatrology and have high value of clinical pathology.

$-C_{2}\left(\right.$ Clinical Features, $\left.w_{2}=0.32\right)$. Represents disease severity, pain and limitations on activity, etc.

- $C_{3}$ (Related risks, $\left.w_{3}=0.20\right)$ Delay could exacerbate a patient's condition or even lead to death, due to infection, comorbidity etc.

- $C_{4}$ (Urgency $w_{4}=0.22$ ) Patients who are seriously ill or have a condition requiring emergency treatment are classified as having high urgency.

- $C_{5}$ (Patient Information, $w_{5}=0.11$ ). Represents medical insurance type, time on waiting list, VIP status (yes or no) etc.

For simplicity of presentation we will use five patients $X=\left\{X_{1}, X_{2}, X_{3}, X_{4}, X_{5}\right\}$ to illustrate the proposed method. We invited hospital experts (such as the outpatient doctor and staff in the admission center) to diagnose these patients and provide their assessments in IMSs. For example, the expert may consider that the patient value (based on admission criteria) is between extreme acceptance and very strong acceptance, so the patient value can be described as a number between 7 and 9. The other values can be deduced in analogous fashion and then we can derive the intuitionistic multiplicative judgment matrix as shown in Table 2.

Table 2 The intuitionistic multiplicative judgment matrix

\begin{tabular}{llllll}
\hline Patients & $C_{1}$ & $C_{2}$ & $C_{3}$ & $C_{4}$ & $C_{5}$ \\
\hline$X_{1}$ & $(6,1 / 8,4 / 3)$ & $(3,1 / 5,5 / 3)$ & $(4,1 / 7,7 / 4)$ & $(1 / 3,2,3 / 2)$ & $(5,1 / 6,6 / 5)$ \\
$X_{2}$ & $(1,1 / 4,4)$ & $(8,1 / 9,9 / 8)$ & $(3,1 / 5,5 / 3)$ & $(1 / 7,2,7 / 2)$ & $(3,1 / 4,4 / 3)$ \\
$X_{3}$ & $(3,1 / 6,2)$ & $(4,1 / 5,5 / 4)$ & $(1,1 / 8,8)$ & $(1 / 5,5,1)$ & $(7,1 / 8,8 / 7)$ \\
$X_{4}$ & $(7,1 / 8,8 / 7)$ & $(3,1 / 6,2)$ & $(5,1 / 6,6 / 5)$ & $(1 / 8,6,4 / 3)$ & $(6,1 / 7,7 / 6)$ \\
$X_{5}$ & $(4,1 / 6,3 / 2)$ & $(7,1 / 8,8 / 7)$ & $(7,1 / 8,8 / 7)$ & $(1 / 6,4,3 / 2)$ & $(5,1 / 9,9 / 5)$ \\
\hline
\end{tabular}

\subsection{Applications of the methods}

As we know, MCDM methods, such as TOPSIS and VIKOR, can be applied to rank options and they are all based on a measure of distance from some reference (ideal) points or options. Below we use the distance-based IM- 
TOPSIS and IM-VIKOR methods to solve this inpatient admission assessment problem.

\subsubsection{Solving the problem with the distance-based IM-TOPSIS method}

Step 1. Derive the intuitionistic multiplicative decision matrix from the values given by the outpatient doctor and the staff in admission service center for clinical and administrative criteria respectively (see Table 1).

Step 2. Determine IMPIS $X^{+}$and IMNIS $X^{-}$. In our example, the criteria $C_{1}, C_{2}, C_{4}$ and $C_{5}$ are benefit criteria while $C_{3}$ is the cost criterion. Thus we get the following IMPIS $A$ and IMNIS $B$.

$$
\begin{gathered}
X^{+}=((7,1 / 8,8 / 7),(8,1 / 9,9 / 8),(1,1 / 8,8),(1 / 3,2,3 / 2),(7,1 / 8,8 / 7)) \\
X^{-}=((1,1 / 4,4),(3,1 / 5,5 / 3),(7,1 / 8,8 / 7),(1 / 8,6,4 / 3),(3,1 / 4,4 / 3))
\end{gathered}
$$

Step 3. The distance between each option and the IMPIS (denoted as PIS-D in Tables 3-5), and the distance between each option and the IMNIS (denoted as NIS-D in Tables 3-5), are given by Eq. (11) and Eq. (16) respectively. The results are shown in Tables 3-4.

Table 3 Distances between each option and the IMPIS or IMNIS for different values of $\lambda$

\begin{tabular}{|c|c|c|c|c|c|c|c|c|c|c|c|c|c|c|}
\hline & \multicolumn{2}{|c|}{$\lambda=1$} & \multicolumn{2}{|c|}{$\lambda=2$} & \multicolumn{2}{|c|}{$\lambda=3$} & \multicolumn{2}{|c|}{$\lambda=4$} & \multicolumn{2}{|c|}{$\lambda=5$} & \multicolumn{2}{|c|}{$\lambda=6$} & \multicolumn{2}{|c|}{$\lambda=7$} \\
\hline & PIS-D & NIS-D & PIS-D & NIS-D & PIS-D & NIS-D & PIS-D & NIS-D & PIS-D & NIS-D & PIS-D & NIS-D & PIS-D & NIS-D \\
\hline$X_{1}$ & 0.1543 & 0.1544 & 0.1867 & 0.188 & 0.2125 & 0.2149 & 0.2317 & 0.2372 & 0.2462 & 0.256 & 0.2573 & 0.272 & 0.2662 & 0.2856 \\
\hline$X_{2}$ & 0.2014 & 0.165 & 0.2334 & 0.1732 & 0.257 & 0.1824 & 0.2768 & 0.1899 & 0.2936 & 0.1959 & 0.308 & 0.2007 & 0.3202 & 0.2045 \\
\hline$X_{3}$ & 0.1253 & 0.1918 & 0.1359 & 0.2306 & 0.144 & 0.2704 & 0.1504 & 0.3006 & 0.1557 & 0.3229 & 0.1603 & 0.3395 & 0.1642 & 0.3523 \\
\hline$X_{4}$ & 0.2166 & 0.1124 & 0.239 & 0.163 & 0.263 & 0.2078 & 0.2832 & 0.2429 & 0.2998 & 0.2699 & 0.3135 & 0.2911 & 0.3249 & 0.3079 \\
\hline \multirow[t]{3}{*}{$X_{5}$} & 0.1635 & 0.1496 & 0.2185 & 0.1586 & 0.2646 & 0.1739 & 0.2977 & 0.1888 & 0.3214 & 0.2019 & 0.3388 & 0.2133 & 0.3519 & 0.223 \\
\hline & \multicolumn{2}{|c|}{$\lambda=8$} & \multicolumn{2}{|c|}{$\lambda=9$} & \multicolumn{2}{|c|}{$\lambda=10$} & \multicolumn{2}{|c|}{$\lambda=20$} & \multicolumn{2}{|c|}{$\lambda=50$} & \multicolumn{2}{|c|}{$\lambda=100$} & \multicolumn{2}{|c|}{$\lambda=400$} \\
\hline & PIS-D & NIS-D & PIS-D & NIS-D & PIS-D & NIS-D & PIS-D & NIS-D & PIS-D & NIS-D & PIS-D & NIS-D & PIS-D & NIS-D \\
\hline$X_{1}$ & 0.2735 & 0.2972 & 0.2795 & 0.307 & 0.2845 & 0.3154 & 0.3105 & 0.3582 & 0.3304 & 0.3871 & 0.338 & 0.3973 & 0.3439 & 0.4051 \\
\hline$X_{2}$ & 0.3306 & 0.2077 & 0.3395 & 0.2104 & 0.3472 & 0.2128 & 0.3894 & 0.2262 & 0.4205 & 0.2392 & 0.4315 & 0.2445 & 0.44 & 0.2486 \\
\hline$X_{3}$ & 0.1676 & 0.3623 & 0.1706 & 0.3704 & 0.1733 & 0.377 & 0.188 & 0.4086 & 0.1996 & 0.4288 & 0.204 & 0.4357 & 0.2074 & 0.441 \\
\hline$X_{4}$ & 0.3344 & 0.3215 & 0.3424 & 0.3328 & 0.3493 & 0.3422 & 0.3855 & 0.389 & 0.4123 & 0.4205 & 0.4219 & 0.4315 & 0.4292 & 0.44 \\
\hline$X_{5}$ & 0.3621 & 0.2313 & 0.3703 & 0.2385 & 0.377 & 0.2447 & 0.4086 & 0.2772 & 0.4288 & 0.2995 & 0.4357 & 0.3074 & 0.441 & 0.3134 \\
\hline
\end{tabular}

(Based on generalized weighted distances)

Table 4 Distances between each option and the IMPIS or IMNIS for different values of $\lambda$

(Based on generalized weighted Hausdorff distances)

\begin{tabular}{clllllllllllllll}
\hline & \multicolumn{2}{c}{$\lambda=1$} & \multicolumn{2}{c}{$\lambda=2$} & \multicolumn{2}{c}{$\lambda=3$} & \multicolumn{2}{c}{$\lambda=4$} & \multicolumn{2}{c}{$\lambda=5$} & \multicolumn{2}{c}{$\lambda=6$} & \multicolumn{2}{c}{$\lambda=7$} & \\
& PIS-D & NIS-D & PIS-D & NIS-D & PIS-D & NIS-D & PIS-D & NIS-D & PIS-D & NIS-D & PIS-D & NIS-D & PIS-D & NIS-D \\
\hline$X_{1}$ & 0.1543 & 0.1544 & 0.2017 & 0.2084 & 0.2282 & 0.2421 & 0.246 & 0.2669 & 0.2591 & 0.286 & 0.2693 & 0.301 & 0.2777 & 0.313 \\
$X_{2}$ & 0.2014 & 0.165 & 0.2592 & 0.1927 & 0.2904 & 0.2035 & 0.312 & 0.2096 & 0.3281 & 0.2137 & 0.3406 & 0.2167 & 0.3506 & 0.2191 \\
\hline
\end{tabular}




\begin{tabular}{|c|c|c|c|c|c|c|c|c|c|c|c|c|c|c|}
\hline$X_{3}$ & 0.1253 & 0.1918 & 0.152 & 0.2378 & 0.1629 & 0.2753 & 0.1693 & 0.3033 & 0.1737 & 0.3243 & 0.177 & 0.3402 & 0.1796 & 0.3526 \\
\hline$X_{4}$ & 0.2166 & 0.1124 & 0.259 & 0.184 & 0.2848 & 0.2385 & 0.3045 & 0.2765 & 0.3207 & 0.3033 & 0.334 & 0.3229 & 0.345 & 0.3377 \\
\hline \multirow[t]{3}{*}{$X_{5}$} & 0.1635 & 0.1496 & 0.2204 & 0.1801 & 0.2653 & 0.1989 & 0.2979 & 0.2133 & 0.3215 & 0.2252 & 0.3388 & 0.2351 & 0.3519 & 0.2434 \\
\hline & \multicolumn{2}{|c|}{$\lambda=8$} & \multicolumn{2}{|c|}{$\lambda=9$} & \multicolumn{2}{|c|}{$\lambda=10$} & \multicolumn{2}{|c|}{$\lambda=20$} & \multicolumn{2}{|c|}{$\lambda=50$} & \multicolumn{2}{|c|}{$\lambda=100$} & \multicolumn{2}{|c|}{$\lambda=400$} \\
\hline & PIS-D & NIS-D & PIS-D & NIS-D & PIS-D & NIS-D & PIS-D & NIS-D & PIS-D & NIS-D & PIS-D & NIS-D & PIS-D & NIS-D \\
\hline$X_{1}$ & 0.2845 & 0.3228 & 0.2902 & 0.3309 & 0.295 & 0.3376 & 0.3191 & 0.3708 & 0.3349 & 0.3926 & 0.3403 & 0.4001 & 0.3142 & 0.3445 \\
\hline$X_{2}$ & 0.3589 & 0.2211 & 0.3657 & 0.2228 & 0.3716 & 0.2243 & 0.4031 & 0.2335 & 0.4263 & 0.2426 & 0.4345 & 0.2462 & 0.4407 & 0.4407 \\
\hline$X_{3}$ & 0.1818 & 0.3625 & 0.1837 & 0.3705 & 0.1854 & 0.3771 & 0.1946 & 0.4086 & 0.2023 & 0.4288 & 0.2054 & 0.4357 & 0.2077 & 0.2077 \\
\hline$X_{4}$ & 0.354 & 0.3493 & 0.3615 & 0.3587 & 0.3678 & 0.3663 & 0.3983 & 0.4027 & 0.418 & 0.4263 & 0.4248 & 0.4345 & 0.3648 & 0.43 \\
\hline$X_{5}$ & 0.3621 & 0.2504 & 0.3703 & 0.2564 & 0.377 & 0.2614 & 0.4086 & 0.2869 & 0.4288 & 0.3037 & 0.4357 & 0.3095 & 0.441 & 0.441 \\
\hline
\end{tabular}

Table 5 Distances between each option and the IMPIS or IMNIS for different values of $\lambda$

(Based on generalized hybrid weighted distances)

\begin{tabular}{|c|c|c|c|c|c|c|c|c|c|c|c|c|c|c|}
\hline & \multicolumn{2}{|c|}{$\lambda=1$} & \multicolumn{2}{|c|}{$\lambda=2$} & \multicolumn{2}{|c|}{$\lambda=3$} & \multicolumn{2}{|c|}{$\lambda=4$} & \multicolumn{2}{|c|}{$\lambda=5$} & \multicolumn{2}{|c|}{$\lambda=6$} & \multicolumn{2}{|c|}{$\lambda=7$} \\
\hline & PIS-D & NIS-D & PIS-D & NIS-D & PIS-D & NIS-D & PIS-D & NIS-D & PIS-D & NIS-D & PIS-D & NIS-D & PIS-D & NIS-D \\
\hline$X_{1}$ & 0.1543 & 0.1544 & 0.1944 & 0.1985 & 0.2207 & 0.2293 & 0.2392 & 0.2534 & 0.2529 & 0.2727 & 0.2637 & 0.2883 & 0.2723 & 0.3012 \\
\hline$X_{2}$ & 0.2014 & 0.165 & 0.2466 & 0.1832 & 0.2747 & 0.1935 & 0.2959 & 0.2005 & 0.3127 & 0.2056 & 0.3263 & 0.2094 & 0.3375 & 0.2126 \\
\hline$X_{3}$ & 0.1253 & 0.1918 & 0.1442 & 0.2343 & 0.154 & 0.2728 & 0.1607 & 0.302 & 0.1656 & 0.3236 & 0.1696 & 0.3399 & 0.1729 & 0.3524 \\
\hline$X_{4}$ & 0.2166 & 0.1124 & 0.2492 & 0.1738 & 0.2743 & 0.2242 & 0.2944 & 0.2613 & 0.3109 & 0.2885 & 0.3246 & 0.309 & 0.3358 & 0.3248 \\
\hline \multirow[t]{3}{*}{$X_{5}$} & 0.1635 & 0.1496 & 0.2195 & 0.1697 & 0.2649 & 0.1872 & 0.2978 & 0.2022 & 0.3214 & 0.2148 & 0.3388 & 0.2255 & 0.3519 & 0.2345 \\
\hline & \multicolumn{2}{|c|}{$\lambda=8$} & \multicolumn{2}{|c|}{$\lambda=9$} & \multicolumn{2}{|c|}{$\lambda=10$} & \multicolumn{2}{|c|}{$\lambda=20$} & \multicolumn{2}{|c|}{$\lambda=50$} & \multicolumn{2}{|c|}{$\lambda=100$} & \multicolumn{2}{|c|}{$\lambda=400$} \\
\hline & PIS-D & NIS-D & PIS-D & NIS-D & PIS-D & NIS-D & PIS-D & NIS-D & PIS-D & NIS-D & PIS-D & NIS-D & PIS-D & NIS-D \\
\hline$X_{1}$ & 0.2794 & 0.3118 & 0.2852 & 0.3207 & 0.2902 & 0.3282 & 0.3154 & 0.3655 & 0.333 & 0.3903 & 0.3394 & 0.3989 & 0.3442 & 0.4055 \\
\hline$X_{2}$ & 0.3467 & 0.2151 & 0.3545 & 0.2173 & 0.3612 & 0.2192 & 0.3973 & 0.2304 & 0.4239 & 0.2412 & 0.4332 & 0.2455 & 0.4404 & 0.2489 \\
\hline$X_{3}$ & 0.1757 & 0.3624 & 0.1781 & 0.3704 & 0.1802 & 0.3771 & 0.1919 & 0.4086 & 0.2012 & 0.4288 & 0.2048 & 0.4357 & 0.2076 & 0.441 \\
\hline$X_{4}$ & 0.3452 & 0.3374 & 0.353 & 0.3476 & 0.3596 & 0.3561 & 0.3929 & 0.397 & 0.4156 & 0.4239 & 0.4236 & 0.4332 & 0.4297 & 0.4404 \\
\hline$X_{5}$ & 0.3621 & 0.2422 & 0.3703 & 0.2487 & 0.377 & 0.2543 & 0.4086 & 0.2828 & 0.4288 & 0.302 & 0.4357 & 0.3086 & 0.441 & 0.3137 \\
\hline
\end{tabular}

Step 4. We can obtain different relative closeness coefficients for different values of parameter $\lambda$ from Eq. (46); the results are shown in Tables 6-8.

Table 6 Patient rankings for different values of $\lambda$

(Based on generalized weighted distances)

\begin{tabular}{lcccccccccccccc}
\hline & $\lambda=1$ & $\lambda=2$ & $\lambda=3$ & $\lambda=4$ & $\lambda=5$ & $\lambda=6$ & $\lambda=7$ & $\lambda=8$ & $\lambda=9$ & $\lambda=10$ & $\lambda=20$ & $\lambda=50$ & $\lambda=100$ & $\lambda=400$ \\
\hline$X_{1}$ & 0.5002 & 0.5017 & 0.5028 & 0.5059 & 0.5098 & 0.5139 & 0.5176 & 0.5208 & 0.5234 & 0.5258 & 0.5357 & 0.5395 & 0.5403 & 0.5409 \\
$X_{2}$ & 0.4503 & 0.4260 & 0.4151 & 0.4069 & 0.4002 & 0.3945 & 0.3897 & 0.3858 & 0.3826 & 0.3800 & 0.3674 & 0.3626 & 0.3617 & 0.3610 \\
$X_{3}$ & 0.6049 & 0.6292 & 0.6525 & 0.6665 & 0.6747 & 0.6793 & 0.6821 & 0.6837 & 0.6847 & 0.6851 & 0.6849 & 0.6824 & 0.6811 & 0.6801 \\
$X_{4}$ & 0.3416 & 0.4055 & 0.4414 & 0.4617 & 0.4738 & 0.4815 & 0.4866 & 0.4902 & 0.4929 & 0.4949 & 0.5023 & 0.5049 & 0.5056 & 0.5062 \\
$X_{5}$ & 0.4778 & 0.4206 & 0.3966 & 0.3881 & 0.3858 & 0.3863 & 0.3879 & 0.3898 & 0.3918 & 0.3936 & 0.4042 & 0.4112 & 0.4137 & 0.4154 \\
$\begin{array}{l}\text { Ranking } \\
\text { orders }\end{array}$ & 31524 & 31254 & 31425 & 31425 & 31425 & 31425 & 31425 & 31452 & 31452 & 31452 & 31452 & 31452 & 31452 & 31452 \\
\hline
\end{tabular}

Table 7 Patient rankings for different values of $\lambda$

(Based on generalized weighted Hausdorff distances)

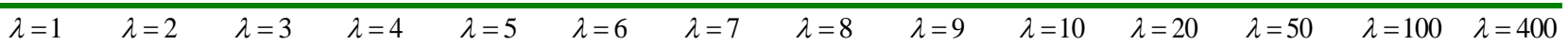




\begin{tabular}{|c|c|c|c|c|c|c|c|c|c|c|c|c|c|c|}
\hline$X_{1}$ & 0.5002 & 0.5082 & 0.5148 & 0.5204 & 0.5247 & 0.5278 & 0.5299 & 0.5315 & 0.5328 & 0.5337 & 0.5375 & 0.5397 & 0.5404 & 0.5409 \\
\hline$X_{2}$ & 0.4503 & 0.4264 & 0.4120 & 0.4018 & 0.3944 & 0.3888 & 0.3846 & 0.3812 & 0.3786 & 0.3764 & 0.3668 & 0.3627 & 0.3617 & 0.3611 \\
\hline$X_{3}$ & 0.6049 & 0.6101 & 0.6283 & 0.6418 & 0.6512 & 0.6578 & 0.6625 & 0.6660 & 0.6685 & 0.6704 & 0.6774 & 0.6794 & 0.6796 & 0.6798 \\
\hline$X_{4}$ & 0.3416 & 0.4153 & 0.4558 & 0.4759 & 0.4861 & 0.4916 & 0.4947 & 0.4967 & 0.4981 & 0.4990 & 0.5027 & 0.5049 & 0.5056 & 0.5061 \\
\hline$X_{5}$ & 0.4778 & 0.4497 & 0.4285 & 0.4173 & 0.4119 & 0.4097 & 0.4089 & 0.4088 & 0.4091 & 0.4095 & 0.4125 & 0.4146 & 0.4153 & 0.4159 \\
\hline $\begin{array}{c}\text { Ranking } \\
\text { orders }\end{array}$ & 31524 & 31524 & 31452 & 31452 & 31452 & 31452 & 31452 & 31452 & 31452 & 31452 & 31452 & 31452 & 31452 & 31452 \\
\hline
\end{tabular}

Table 8 Patient rankings for different values of $\lambda$

(Based on generalized hybrid weighted distances)

\begin{tabular}{|c|c|c|c|c|c|c|c|c|c|c|c|c|c|c|}
\hline & $\lambda=1$ & $\lambda=2$ & $\lambda=3$ & $\lambda=4$ & $\lambda=5$ & $\lambda=6$ & $\lambda=7$ & $\lambda=8$ & $\lambda=9$ & $\lambda=10$ & $\lambda=20$ & $\lambda=50$ & $\lambda=100$ & $\lambda=400$ \\
\hline$X_{1}$ & 0.5002 & 0.5052 & 0.5096 & 0.5144 & 0.5188 & 0.5223 & 0.5252 & 0.5274 & 0.5293 & 0.5307 & 0.5368 & 0.5396 & 0.5403 & 0.5409 \\
\hline$X_{2}$ & 0.4503 & 0.4262 & 0.4133 & 0.4039 & 0.3967 & 0.3909 & 0.3865 & 0.3829 & 0.3800 & 0.3777 & 0.3671 & 0.3627 & 0.3617 & 0.3611 \\
\hline$X_{3}$ & 0.6049 & 0.6190 & 0.6392 & 0.6527 & 0.6615 & 0.6671 & 0.6709 & 0.6735 & 0.6753 & 0.6767 & 0.6804 & 0.6806 & 0.6802 & 0.6799 \\
\hline$X_{4}$ & 0.3416 & 0.4109 & 0.4497 & 0.4702 & 0.4813 & 0.4877 & 0.4917 & 0.4943 & 0.4961 & 0.4976 & 0.5026 & 0.5049 & 0.5056 & 0.5061 \\
\hline$X_{5}$ & 0.4778 & 0.4360 & 0.4141 & 0.4044 & 0.4006 & 0.3996 & 0.3999 & 0.4008 & 0.4018 & 0.4028 & 0.4090 & 0.4132 & 0.4146 & 0.4157 \\
\hline $\begin{array}{l}\text { Ranking } \\
\text { orders }\end{array}$ & 31524 & 31524 & 31425 & 31425 & 31452 & 31452 & 31452 & 31452 & 31452 & 31452 & 31452 & 31452 & 31452 & 31452 \\
\hline
\end{tabular}

Step 5. The relative closeness coefficient values are sorted in descending order, as shown in the final rows of Tables 6-8. Note that in these tables the notation "31524" represents the following ranking order: $X_{3} \succ X_{1} \succ X_{5} \succ X_{2} \succ X_{4}$

Step 6. Rank all patients in ascending order of the values of $I M G U_{i}, I M I R_{i}$ and $I M C_{i}$ and propose compromise solution(s) for the MCDM problem, based on which the ranking list can be derived. The final rankings with respect to various parameters are shown in Tables 6-8.

\subsubsection{Solving the problem with the distance-based IM-VIKOR method}

Steps 1-2. As described in 5.2.1.

Step 3. Calculate the values of $I M G U_{i}$ and $I M I R_{i}$ for all patients with Eq. (47) and Eq. (48), respectively. The results are shown in Table 9.

Table 9 The values of $I M G U_{i}$ and $I M I R_{i}$

\begin{tabular}{cccccc}
\hline & $X_{1}$ & $X_{2}$ & $X_{3}$ & $X_{4}$ & $X_{5}$ \\
\hline$I M G U_{i}$ & 0.5615 & 0.7031 & 0.4530 & 0.6550 & 0.5149 \\
$I M I R_{i}$ & 0.3013 & 0.3278 & 0.228 & 0.3009 & 0.2487 \\
\hline
\end{tabular}

Step 4. Calculate the values of $I M C_{i}$ for all patients using Eq. (49). The results are shown in Table 10.

Table 10 Patient rankings for different values of parameter $v$

\begin{tabular}{ccccccc}
\hline$v$ & $I M C_{1}$ & $I M C_{2}$ & $I M C_{3}$ & $I M C_{4}$ & $I M C_{5}$ & Ranking orders \\
\hline 0.0 & 0.7345 & 1 & 0 & 0.7305 & 0.2074 & 35412 \\
0.1 & 0.7044 & 1 & 0 & 0.7382 & 0.2114 & 35142 \\
0.2 & 0.6743 & 1 & 0 & 0.7459 & 0.2154 & 35142 \\
\hline
\end{tabular}




\begin{tabular}{lcllcll}
\hline 0.3 & 0.6443 & 1 & 0 & 0.7536 & 0.2194 & 35142 \\
0.4 & 0.6142 & 1 & 0 & 0.7613 & 0.2234 & 35142 \\
0.5 & 0.5841 & 1 & 0 & 0.7691 & 0.2275 & 35142 \\
0.6 & 0.5541 & 1 & 0 & 0.7768 & 0.2315 & 35142 \\
0.7 & 0.524 & 1 & 0 & 0.7845 & 0.2355 & 35142 \\
0.8 & 0.494 & 1 & 0 & 0.7922 & 0.2395 & 35142 \\
0.9 & 0.4639 & 1 & 0 & 0.8 & 0.2435 & 35142 \\
\hline
\end{tabular}

Steps 5-6. Rank all patients in ascending order of the values of $I M G U_{i}, I M I R_{i}$ and $I M C_{i}$ and propose the compromise solution(s) for the MCDM problem. The final rankings for different parameter values are shown in Table 10.

\subsection{Analyses of results}

We can derive some interesting conclusions from Tables 7-9.

(1) In the case of the IM-TOPSIS method, we use generalized weighted distance, generalized weighted Hausdorff distance and generalized hybrid weighted distance to calculate the corresponding distances, respectively. These different distance measures produce different relative closeness coefficient results, but produced similar ranking results, as listed in Tables 7-9. When $\lambda=2$, the ranking result is $X_{3} \succ X_{1} \succ X_{2} \succ X_{5} \succ X_{4}$ or $X_{3} \succ X_{1} \succ X_{5} \succ X_{2} \succ X_{4}$. However, if $\lambda \geq 3$, the rankings are all $X_{3} \succ X_{1} \succ X_{4} \succ X_{5} \succ X_{2}$. In such cases, ranking result does not change as the value of $\lambda$ increase, regardless of whether generalized weighted distance, generalized weighted Hausdorff distance or generalized hybrid weighted distance is used. In addition, the results derived from these three distance measures become more stable as parameter $\lambda$ increases. This result is consistent with the conclusion presented in Section 3.

(2) The variability of the values of the relative closeness coefficients differs with respect to $\lambda$. For $X_{2}$ calculated from the above three distance measures, the values of the relative closeness coefficients decrease as parameter $\lambda$ increases. For $X_{1}$ and $X_{4}$, the variability in relative closeness coefficients follows a different pattern from that applying to $X_{2}$, which always increases as parameter $\lambda$ increases. For $X_{3}$ and $X_{5}$, however, the variation tendencies of the values of the relative closeness coefficients not always the same.

As mentioned above, using the IM-TOPSIS method, the relative closeness coefficient slightly varies with the selected distance measures for IMSs, including the generalized weighted distance, generalized weighted Hausdorff distance and generalized hybrid weighted distance. However, when we consider the values of the relative closeness 
coefficient from the perspective of final results, we find that they converge on an instant number, such as 0.5409 , $0.3610,0.6801,0.5062$ and 0.4154 in Table 7 . Therefore, in real case, the DMs only need to compute the generalized hybrid distance measures of IMS with $\lambda=1$ or 2 . In addition, as an integrated form of the Minkowski distance and Hausdorff distance, the generalized hybrid weighted distance measure for IMS has stronger robustness than the generalized weighted form.

Using the IM-VIKOR method we can calculate distances using the Euclidean distance measure and obtain some meaningful results. When $v$ is changed, the rankings also change. For example, when $v=0$, the rank order is $X_{3} \succ X_{5} \succ X_{4} \succ X_{1} \succ X_{2}$. However, if $v \neq 0$, the rank order becomes $X_{3} \succ X_{5} \succ X_{1} \succ X_{4} \succ X_{2}$, based on which, the compromise solution(s) can be derived as $X_{3}$ and $X_{5}$. The DMs can determine the value of $v$ and we should note that $v$ is the weight of the decision making strategy "the majority of criteria", or saying "the maximum group utility" (Opricovic \& Tzeng, 2004), based on the DMs' preference in the given MCDM problem. The VIKOR method is more useful and flexible in our real-life situation because it allows the DMs to try to both maximize group utility and minimize individual regret of other DMs (opponents).

Analysis of our example case shows that both the IM-TOPSIS method and the IM-VIKOR method can handle the inpatient admission assessment problem effectively. The results explain the difference between these two MCDM methods. It also shows that the IM-TOPSIS method and the IM-VIKOR method produce different rankings when dealing with the inpatient admission assessment problem. Using the IM-TOPSIS method the best ranking is $X_{3} \succ X_{1} \succ X_{4} \succ X_{5} \succ X_{2}$, whilst using the IM-VIKOR method the compromise ranking result is $X_{3} \succ X_{5} \succ X_{1} \succ X_{4} \succ X_{2}$. In real life, DMs can choose any one of them, the IM-TOPSIS or the IM-VIKOR method, according to the characteristics of the actual inpatient admission assessment problem.

\section{Conclusions}

As we know, distance measures are a widely used to deal with MCDM problems. In this paper we have developed some distance measures for IMSs, including the generalized Hausdorff distance, weighted distance measures, ordered weighted distance measures and continuous weighted distance measures. On this basis, we have proposed a distance-based IM-TOPSIS method and a distance-based IM-VIKOR method, which can be used to handle MCDM problems with IMSs. Compared with the IM-VIKOR method, the IM-TOPSIS method produces more stable and accurate results when used to handle an admission-sequencing problem. We have used a practical 
example in the field of hospital management to illustrate these proposed approaches. As to the studied case of inpatient admission service center in $\mathrm{WCH}$, we have proposed the evaluation indices, or saying criteria, for the admission sequencing model. It can provide some references for the hospital managers in China.

In the future we may investigate the differences between generalized continue weighted distance and generalized ordered weighted distance as applied to IMSs. The issue of how to handle MCDM problems when criterion weights are unknown or incomplete remains open. We will try to develop more measures of the distance between IMSs, for example projection-based distance measures, psychological distance measures etc. Furthermore, we may apply our distance measures to other decision-making problems.

\section{Acknowledgements}

The work was supported by the National Natural Science Foundation of China $(71971145,71771156$, 71532007).

\section{Conflicts of interest}

None.

\section{References}

Ashour, O. M., \& Kremer, G. E. O. (2016). Dynamic patient grouping and prioritization: a new approach to emergency department flow improvement. Health Care Management Science, 19(2), 192-205. doi: 10.1007/s10729-014-9311-1

Atanassov, K. T. (1986). Intuitionistic fuzzy sets. Fuzzy Sets and Systems, 20(1), 87-96.

Bausys, R., \& Zavadskas, E. K. (2015). Multi-criteria decision making approach by VIKOR under interval neutrosophic set environment. Economic Computation and Economic Cybernetics Studies and Research, 49(4), $33-48$.

Burillo, P., \& Bustince, H. (1996). Entropy on intuitionistic fuzzy sets and on interval-valued fuzzy sets. Fuzzy Sets and Systems, 78(30), 305-316. doi: https://doi.org/10.1016/0165-0114(96)84611-2

Chen, S. J., \& Hwang, C. L. (1992). Fuzzy Multiple Attribute Decision Making: Methods and Applications. Springer-Verlag, Berlin.

Du, W. S., \& Hu, B. Q. (2015). Aggregation distance measure and its induced similarity measure between intuitionistic fuzzy sets. Pattern Recognition Letters, 60(C), 65-71. doi: https://doi.org/10.1016/j.patrec.2015.03.001 
Garg, H. (2017). Distance and similarity measures for intuitionistic multiplicative preference relation and its applications. International Journal for Uncertainty Quantification, 7(2), 117-133. doi: 10.1615/Int.J.UncertaintyQuantification.2017018981

Grzegorzewski, P. (2004). Distances between intuitionistic fuzzy sets and/or interval-valued fuzzy sets based on the Hausdorff metric. Fuzzy Sets and Systems, 148(2), 319-328. doi: https://doi.org/10.1016/j.fss.2003.08.005

Jiang, Y., Xu, Z. S., \& Gao, M. (2015). Methods for ranking intuitionistic multiplicative numbers by distance measures in decision making. Computers \& Industrial Engineering, 88(C), 100-109. doi: https://doi.org/10.1016/j.cie.2015.06.015

Jiang, Y., Xu, Z. S., \& Shu, Y. H. (2016). Distance measures between intuitionistic multiplicative sets and their application in site selection of building satellite earth stations. Systems Engineering-Theory and Practice, 36(12), 3210-3219. doi: 10.12011/1000-6788(2016)12-3210-10

Jiang, Y., Xu, Z. S., \& Yu, X. H. (2013). Compatibility measures and consensus models for group decision making with intuitionistic multiplicative preference relations. Applied Soft Computing, 13(4), 2075-2086. doi: https://doi.org/10.1016/j.asoc.2012.11.007

Jiang, Y., Xu, Z. S., \& Yu, X. H. (2015). Group decision making based on incomplete intuitionistic multiplicative preference relations. Information Sciences, 295, 33-52. doi: https://doi.org/10.1016/j.ins.2014.09.043

Liao, H. C., \& Xu, Z. S. (2013). A VIKOR-based method for hesitant fuzzy multi-criteria decision making. Fuzzy Optimization and Decision Making, 12(4), 373-392. doi: 10.1007/s10700-013-9162-0

Liao, H. C., \& Xu, Z. S. (2015). Approaches to manage hesitant fuzzy linguistic information based on the cosine distance and similarity measures for HFLTSs and their application in qualitative decision making. Expert Systems with Applications, 42(12), 5328-5336. doi: https://doi.org/10.1016/j.eswa.2015.02.017

Liao, H. C., Xu, Z. S., \& Zeng, X. J. (2014). Distance and similarity measures for hesitant fuzzy linguistic term sets and their application in multi-criteria decision making. Information Sciences, 271, 125-142. doi: https://doi.org/10.1016/j.ins.2014.02.125

Liao, H. C., Xu, Z. S., \& Zeng, X. J. (2015). Hesitant fuzzy linguistic VIKOR method and its application in qualitative multiple criteria decision making. IEEE Transactions on Fuzzy Systems, 23(5), 1343-1355. doi: 10.1109/TFUZZ.2014.2360556

Liu W. S., \& Liao, H. C. (2017). A bibliometric analysis of fuzzy decision research during 1970-2015. International Journal of Fuzzy Systems, 19(1), 1-14. doi: 10.1007/s40815-016-0272-z

Luo, L., Zhang, C., \& Liao, H. C. (2019). Distance-based intuitionistic multiplicative MULTIMOORA method integrating a novel weight-determining method for multiple criteria group decision making. Computers and Industrial Engineering, 131, 82-98. doi: https://doi.org/10.1016/j.cie.2019.03.038

Mardani, A., Zavadskas, E. K., Govindan, K., Senin, A. A., \& Jusoh, A. (2016). VIKOR technique: a systematic review of the state of the art literature on methodologies and applications. Sustainability, 8(37), 1-38. doi: https://doi.org/10.3390/su8010037 
Mariotti, G., Siciliani, L., Rebba, V., et al. (2014). Waiting time prioritisation for specialist services in Italy: The homogeneous waiting time groups approach. Health Policy, 117(1), 54-63. doi: https://doi.org/10.1016/j.healthpol.2014.01.018

Narukawa, Y., \& Torra, V. (2006). Non-monotonic fuzzy measures and intuitionistic fuzzy sets. International Conference on Modeling Decisions for Artificial Intelligence, Springer-Verlag, 150-160.

Opricovic, S. (1998). Multicriteria Optimization of Civil Engineering Systems. Faculty of Civil Engineering, Belgrade. 2(1), 5-21.

Opricovic, S., \& Tzeng, G. H. (2004). Compromise solution by MCDM methods: A comparative analysis of VIKOR and TOPSIS. European Journal of Operational Research, 156(2), 445-455. doi: https://doi.org/10.1016/S0377-2217(03)00020-1

Rahimi, S. A., Jamshidi, A., Ait-Kadi, D., \& Bartolome, A. R. (2015). Risk-based decision making framework for prioritizing patients' access to healthcare services by considering uncertainties. International Conference on Industrial Engineering and Systems Management, IEEE, 291-297. doi: 10.1109/IESM.2015.7380173

Rahimi, S. A., Jamshidi, A., Ruiz, A., Ait-kadi, D. (2016). A new dynamic integrated framework for surgical patients' prioritization considering risks and uncertainties. Decision Support Systems, 88, 112-120. doi: https://doi.org/10.1016/j.dss.2016.06.003

Ren, P. J., Xu, Z. S., \& Liao, H. C. (2016). Intuitionistic multiplicative analytic hierarchy process in group decision making. Computers and Industrial Engineering, 101, 513-524. doi: https://doi.org/10.1016/j.cie.2016.09.025

Saaty, T. L. (1990). How to make a decision: The analytic hierarchy process. European Journal of Operational Research, 48(1), 9-26. doi: https://doi.org/10.1016/0377-2217(90)90057-I

Solans-Domènech, M., Adam, P., Tebé, C., \& Espallargues, M. (2013). Developing a universal tool for the prioritization of patients waiting for elective surgery. Health Policy, 113(1), 118-126. doi: https://doi.org/10.1016/j.healthpol.2013.07.006

Szmidt, E., \& Kacprzyk, J. (2000). Distances between intuitionistic fuzzy sets. Fuzzy Sets and Systems, 114(3), 505-518. doi: https://doi.org/10.1016/S0165-0114(98)00244-9

Valente, R., Testi, A., Tanfani, E., Fato, M., Porro, I., Santo, M., Santori, G., Torre, G., \& Ansaldo, G. (2009). A model to prioritize access to elective surgery on the basis of clinical urgency and waiting time. BMC Health Services Research, 9(1), 1-15. doi: 10.1186/1472-6963-9-1

Wang, W., \& Xin, X. (2005). Distance measure between intuitionistic fuzzy sets. Pattern Recognition Letters, 26(13), 2063-2069. doi: https://doi.org/10.1016/j.patrec.2005.03.018

Xu, Z. S. (2008). Hybrid weighted distance measures and their application to pattern recognition. Lecture Notes in Computer Science, 5326, 17-23.

Xu, Z. S. (2012). Fuzzy ordered distance measures. Fuzzy Optimization and Decision Making, 11(1), 73-97. doi: $10.1007 / \mathrm{s} 10700-011-9113-6$

Xu, Z. S. (2013). Priority weight intervals derived from intuitionistic multiplicative preference relations. IEEE 
Transactions on Fuzzy Systems, 21(4), 642-654. doi: 10.1109/TFUZZ.2012.2226893

Xu, Z. S., \& Chen, J. (2008). Ordered weighted distance measure. Journal of Systems Science and Systems Engineering, 17(4), 432-445. doi: 10.1007/s11518-008-5084-8

Xu, Z. S., \& Chen, J. (2011). An overview of distance and similarity measures of intuitionistic fuzzy sets. International Journal of Uncertainty Fuzziness and Knowledge-Based Systems, 16(4), 529-555. doi: https://doi.org/10.1142/S0218488508005406

Xu, Z. S., \& Xia, M. M. (2011). Distance and similarity measures for hesitant fuzzy sets. Information Sciences, 181(11), 2128-2138. doi: https://doi.org/10.1016/j.ins.2011.01.028

Xia, M. M., Xu, Z. S., \& Liao, H. C. (2013). Preference relations based on intuitionistic multiplicative information. IEEE Transactions on Fuzzy Systems, 21(1) 113-133. doi: 10.1109/TFUZZ.2012.2202907

Yu, D. J., \& Liao, H. C. (2016). Visualization and quantitative research on intuitionistic fuzzy studies. Journal of Intelligent \& Fuzzy Systems, 30(6), 3653-3663. doi: 10.3233/IFS-162111

Zhang, C., Liao, H.C., \& Luo, L. (2019). Additive consistency-based priority-generating method of q-rung orthopair fuzzy preference relation. International Journal of Intelligent Systems, 34(9), 2151-2176. doi: https://doi.org/10.1002/int.22137

Zhang, F. Y., Luo, L., Liao, H. C., Zhu, T., Shi, Y. K., \& Shen, W. W. (2016). Inpatient admission assessment in West China Hospital based on hesitant fuzzy linguistic VIKOR method. Journal of Intelligent and Fuzzy Systems, 30(6), 3143-3154. doi: 10.3233/IFS-152056

Zhang, Z. M., \& Pedrycz, W. (2017). Models of mathematical programming for intuitionistic multiplicative preference relations. IEEE Transactions on Fuzzy Systems, 25(4), 945-957. doi: 10.1109/TFUZZ.2016.2587326

Zhang, Z. M., \& Pedrycz, W. (2018). Intuitionistic multiplicative group analytic hierarchy process and its use in multicriteria group decision-making. IEEE Transactions on Cybernetics, 48(7), 1950-1962. doi: 10.1109/TCYB.2017.2720167

\section{Appendix}

\section{Proof of Property 1.}

Proof. (1) Necessary: If $A=B$, then the equality $\max \left\{\left|\log _{9} \frac{\rho_{A}\left(x_{i}\right)}{\rho_{B}\left(x_{i}\right)}\right|^{\lambda},\left|\log _{9} \frac{\sigma_{A}\left(x_{i}\right)}{\sigma_{B}\left(x_{i}\right)}\right|^{\lambda},\left|\log _{9} \frac{\tau_{A}\left(x_{i}\right)}{\tau_{B}\left(x_{i}\right)}\right|^{\lambda}\right\}=0$ holds. On this basis, we have $\left(\frac{1}{2^{\lambda}} \sum_{i=1}^{n} \max \left\{\left|\log _{9} \frac{\rho_{A}\left(x_{i}\right)}{\rho_{B}\left(x_{i}\right)}\right|^{\lambda},\left|\log _{9} \frac{\sigma_{A}\left(x_{i}\right)}{\sigma_{B}\left(x_{i}\right)}\right|^{\lambda},\left|\log _{9} \frac{\tau_{A}\left(x_{i}\right)}{\tau_{B}\left(x_{i}\right)}\right|^{\lambda}\right\}\right)^{1 / \lambda}=0$. Thus, the equality $d_{\text {gwhaud }}(A, B)=0$ holds. 
Sufficient:

Since

$$
\max \left\{\left|\log _{9} \frac{\rho_{A}\left(x_{i}\right)}{\rho_{B}\left(x_{i}\right)}\right|^{2},\left.\log _{9} \frac{\sigma_{A}\left(x_{i}\right)}{\sigma_{B}\left(x_{i}\right)}\right|^{2},\left|\log _{9} \frac{\tau_{A}\left(x_{i}\right)}{\tau_{B}\left(x_{i}\right)}\right|\right\} \geq 0
$$

$\left(\frac{1}{2^{\lambda}} \sum_{i=1}^{n} \max \left\{\left|\log _{9} \frac{\rho_{A}\left(x_{i}\right)}{\rho_{B}\left(x_{i}\right)}\right|^{\lambda},\left|\log _{9} \frac{\sigma_{A}\left(x_{i}\right)}{\sigma_{B}\left(x_{i}\right)}\right|^{\lambda},\left|\log _{9} \frac{\tau_{A}\left(x_{i}\right)}{\tau_{B}\left(x_{i}\right)}\right|^{\lambda}\right\}\right\}^{1 / \lambda} \geq 0$. If $d_{\text {gwhaud }}(A, B)=\left(\frac{1}{2^{\lambda}} \sum_{i=1}^{n} \max \left\{\left|\log _{9} \frac{\rho_{A}\left(x_{i}\right)}{\rho_{B}\left(x_{i}\right)}\right|^{\lambda},\left|\log _{9} \frac{\sigma_{A}\left(x_{i}\right)}{\sigma_{B}\left(x_{i}\right)}\right|^{\lambda},\left|\log _{9} \frac{\tau_{A}\left(x_{i}\right)}{\tau_{B}\left(x_{i}\right)}\right|^{\lambda}\right\}^{1 / \lambda}\right\}^{1 / \lambda}=0$, then $\quad \max \left\{\left|\log _{9} \frac{\rho_{A}\left(x_{i}\right)}{\rho_{B}\left(x_{i}\right)}\right|^{\lambda},\left|\log _{9} \frac{\sigma_{A}\left(x_{i}\right)}{\sigma_{B}\left(x_{i}\right)}\right|^{\lambda},\left|\log _{9} \frac{\tau_{A}\left(x_{i}\right)}{\tau_{B}\left(x_{i}\right)}\right|\right\}=0 \quad$. On $\quad$ this basis, we can show that $\left|\log _{9} \frac{\rho_{A}\left(x_{i}\right)}{\rho_{B}\left(x_{i}\right)}\right|^{\lambda}=\left|\log _{9} \frac{\sigma_{A}\left(x_{i}\right)}{\sigma_{B}\left(x_{i}\right)}\right|^{\lambda}=\left|\log _{9} \frac{\tau_{A}\left(x_{i}\right)}{\tau_{B}\left(x_{i}\right)}\right|^{\lambda}=0$ holds, namely, $A=B$.

(2) It is apparent that (2) holds and thus the proof is omitted here.

(3) Since

$d_{\text {ghaud }}(A, B)=\left(\frac{1}{2^{\lambda}} \sum_{i=1}^{n} \max \left\{\left|\log _{9} \frac{\rho_{A}\left(x_{i}\right)}{\rho_{B}\left(x_{i}\right)}\right|^{\lambda},\left|\log _{9} \frac{\sigma_{A}\left(x_{i}\right)}{\sigma_{B}\left(x_{i}\right)}\right|^{\lambda},\left|\log _{9} \frac{\tau_{A}\left(x_{i}\right)}{\tau_{B}\left(x_{i}\right)}\right|^{\lambda}\right\}\right)^{1 / \lambda}$

$=\left(\frac{1}{2^{\lambda}} \sum_{i=1}^{n} \max \left\{\left|\log _{9} \rho_{A}\left(x_{i}\right)-\log _{9} \rho_{B}\left(x_{i}\right)\right|^{\lambda},\left|\log _{9} \sigma_{A}\left(x_{i}\right)-\log _{9} \sigma_{B}\left(x_{i}\right)\right|^{\lambda},\left|\log _{9} \tau_{A}\left(x_{i}\right)-\log _{9} \tau_{B}\left(x_{i}\right)\right|^{\lambda}\right\}\right)^{1 / \lambda}$,

for three IMSs $A \subseteq B \subseteq C \quad$, we have $\left|\log _{9} \rho_{A}\left(x_{i}\right)-\log _{9} \rho_{C}\left(x_{i}\right)\right| \geq\left|\log _{9} \rho_{A}\left(x_{i}\right)-\log _{9} \rho_{B}\left(x_{i}\right)\right|$, $\left|\log _{9} \sigma_{A}\left(x_{i}\right)-\log _{9} \sigma_{C}\left(x_{i}\right)\right| \geq\left|\log _{9} \sigma_{A}\left(x_{i}\right)-\log _{9} \sigma_{B}\left(x_{i}\right), \quad\right| \log _{9} \tau_{A}\left(x_{i}\right)-\log _{9} \tau_{C}\left(x_{i}\right)|\geq| \log _{9} \tau_{A}\left(x_{i}\right)-\log _{9} \tau_{B}\left(x_{i}\right) \mid$. It is easy to obtain that $\left(\frac{1}{2^{\lambda}} \sum_{i=1}^{n} \max \left\{\left|\log _{9} \rho_{A}\left(x_{i}\right)-\log _{9} \rho_{C}\left(x_{i}\right)\right|^{\lambda},\left|\log _{9} \sigma_{A}\left(x_{i}\right)-\log _{9} \sigma_{C}\left(x_{i}\right)\right|^{\lambda},\left|\log _{9} \tau_{A}\left(x_{i}\right)-\log _{9} \tau_{C}\left(x_{i}\right)\right|^{\lambda}\right\}\right)^{1 / \lambda} \geq$ $\left(\frac{1}{2^{\lambda}} \sum_{i=1}^{n} \max \left\{\left|\log _{9} \rho_{A}\left(x_{i}\right)-\log _{9} \rho_{B}\left(x_{i}\right)\right|^{\lambda},\left|\log _{9} \sigma_{A}\left(x_{i}\right)-\log _{9} \sigma_{B}\left(x_{i}\right)\right|^{\lambda},\left|\log _{9} \tau_{A}\left(x_{i}\right)-\log _{9} \tau_{B}\left(x_{i}\right)\right|^{\lambda}\right\}\right)^{1 / \lambda}$. Thus, we have $d_{\text {ghaud }}(A, C) \geq d_{\text {ghaud }}(A, B)$. Similarly, we can prove $d_{\text {ghaud }}(A, C) \geq d_{\text {ghaud }}(B, C)$ holds. The proof of Property 1 is completed.

\section{Proof of Property 2.}

Proof. (1) Necessary: If $A=B$, then $\left|\log _{9} \frac{\rho_{A}\left(x_{i}\right)}{\rho_{B}\left(x_{i}\right)}\right|^{\lambda}+\left|\log _{9} \frac{\sigma_{A}\left(x_{i}\right)}{\sigma_{B}\left(x_{i}\right)}\right|^{\lambda}+\left|\log _{9} \frac{\tau_{A}\left(x_{i}\right)}{\tau_{B}\left(x_{i}\right)}\right|^{\lambda}=0$. It is easy to get $\left(\sum_{i=1}^{n}\left(\frac{1}{2^{\lambda+2}}\left(\left|\log _{9} \frac{\rho_{A}\left(x_{i}\right)}{\rho_{B}\left(x_{i}\right)}\right|^{\lambda}+\left|\log _{9} \frac{\sigma_{A}\left(x_{i}\right)}{\sigma_{B}\left(x_{i}\right)}\right|^{\lambda}+\left|\log _{9} \frac{\tau_{A}\left(x_{i}\right)}{\tau_{B}\left(x_{i}\right)}\right|^{\lambda}\right)+\frac{1}{2^{\lambda+1}} \max \left\{\left|\log _{9} \frac{\rho_{A}\left(x_{i}\right)}{\rho_{B}\left(x_{i}\right)}\right|^{\lambda},\left|\log _{9} \frac{\sigma_{A}\left(x_{i}\right)}{\sigma_{B}\left(x_{i}\right)}\right|^{2},\left|\log _{9} \frac{\tau_{A}\left(x_{i}\right)}{\tau_{B}\left(x_{i}\right)}\right|\right\}\right)\right)^{1 / \lambda}=0$. Thus, $d_{g h d}(A, B)=0$.

Sufficient: 
Since $d_{g h d}(A, B)=\left(\sum_{i=1}^{n}\left(\frac{1}{2^{\lambda+2}}\left(\left|\log _{9} \frac{\rho_{A}\left(x_{i}\right)}{\rho_{B}\left(x_{i}\right)}\right|^{2}+\left|\log _{9} \frac{\sigma_{A}\left(x_{i}\right)}{\sigma_{B}\left(x_{i}\right)}\right|^{\lambda}+\left|\log _{9} \frac{\tau_{A}\left(x_{i}\right)}{\tau_{B}\left(x_{i}\right)}\right|^{\lambda}\right)+\frac{1}{2^{\lambda+1}} \max \left\{\left|\log _{9} \frac{\rho_{A}\left(x_{i}\right)}{\rho_{B}\left(x_{i}\right)}\right|^{\lambda}\left|\log _{9} \frac{\sigma_{A}\left(x_{i}\right)}{\sigma_{B}\left(x_{i}\right)}\right|^{\lambda},\left|\log _{9} \frac{\tau_{A}\left(x_{i}\right)}{\tau_{B}\left(x_{i}\right)}\right|^{\lambda}\right\}\right)\right)^{1 / \lambda} \geq 0$, we have $\sum_{i=1}^{n}\left(\frac{1}{2^{\lambda+2}}\left(\left|\log _{9} \frac{\rho_{A}\left(x_{i}\right)}{\rho_{B}\left(x_{i}\right)}\right|^{\lambda}+\left|\log _{9} \frac{\sigma_{A}\left(x_{i}\right)}{\sigma_{B}\left(x_{i}\right)}\right|^{\lambda}+\left|\log _{9} \frac{\tau_{A}\left(x_{i}\right)}{\tau_{B}\left(x_{i}\right)}\right|^{\lambda}\right)=0\right.$ and $\frac{1}{2^{\lambda+1}} \max \left\{\left|\log _{9} \frac{\rho_{A}\left(x_{i}\right)}{\rho_{B}\left(x_{i}\right)}\right|^{\lambda},\left|\log _{9} \frac{\sigma_{A}\left(x_{i}\right)}{\sigma_{B}\left(x_{i}\right)}\right|^{\lambda},\left|\log _{9} \frac{\tau_{A}\left(x_{i}\right)}{\tau_{B}\left(x_{i}\right)}\right|^{\lambda}\right\}=0$. It is easy to show that $\left|\log _{9} \frac{\rho_{A}\left(x_{i}\right)}{\rho_{B}\left(x_{i}\right)}\right|^{\lambda}=0,\left|\log _{9} \frac{\sigma_{A}\left(x_{i}\right)}{\sigma_{B}\left(x_{i}\right)}\right|^{\lambda}=0$, and $\left|\log _{9} \frac{\tau_{A}\left(x_{i}\right)}{\tau_{B}\left(x_{i}\right)}\right|^{\lambda}=0$. Thus, $A=B$ holds.

(2) It is obviously that (2) holds and therefore the proof is omitted here.

(3) Since

$d_{g h d}(A, B)=\left(\sum_{i=1}^{n}\left(\frac{1}{2^{\lambda+2}}\left(\left|\log _{9} \frac{\rho_{A}\left(x_{i}\right)}{\rho_{B}\left(x_{i}\right)}\right|^{\lambda}+\left|\log _{9} \frac{\sigma_{A}\left(x_{i}\right)}{\sigma_{B}\left(x_{i}\right)}\right|^{\lambda}+\left|\log _{9} \frac{\tau_{A}\left(x_{i}\right)}{\tau_{B}\left(x_{i}\right)}\right|^{\lambda}\right)+\frac{1}{2^{\lambda+1}} \max \left\{\left|\log _{9} \frac{\rho_{A}\left(x_{i}\right)}{\rho_{B}\left(x_{i}\right)}\right|^{\lambda},\left|\log _{9} \frac{\sigma_{A}\left(x_{i}\right)}{\sigma_{B}\left(x_{i}\right)}\right|^{\lambda}\left|\log _{9} \frac{\tau_{A}\left(x_{i}\right)}{\tau_{B}\left(x_{i}\right)}\right|^{\lambda}\right\}\right)\right\}^{1 / \lambda}$

$=\left(\sum_{i=1}^{n}\left(\frac{1}{2^{\lambda+2}}\left(\left|\log _{9} \rho_{A}\left(x_{i}\right)-\log _{9} \rho_{B}\left(x_{i}\right)\right|^{\lambda}+\left|\log _{9} \sigma_{A}\left(x_{i}\right)-\log _{9} \sigma_{B}\left(x_{i}\right)\right|^{\lambda}+\left|\log _{9} \tau_{A}\left(x_{i}\right)-\log _{9} \tau_{B}\left(x_{i}\right)\right|^{\lambda}\right)+\right.\right.$

$\left.\frac{1}{2^{\lambda+1}} \max \left\{\left|\log _{9} \rho_{A}\left(x_{i}\right)-\log _{9} \rho_{B}\left(x_{i}\right)\right|^{\lambda},\left|\log _{9} \sigma_{A}\left(x_{i}\right)-\log _{9} \sigma_{B}\left(x_{i}\right)\right|^{\lambda},\left|\log _{9} \tau_{A}\left(x_{i}\right)-\log _{9} \tau_{B}\left(x_{i}\right)\right|^{\lambda}\right\}\right)^{1 / \lambda}$

for three IMSs $A \subseteq B \subseteq C \quad$, we have $\left|\log _{9} \rho_{A}\left(x_{i}\right)-\log _{9} \rho_{C}\left(x_{i}\right)\right| \geq\left|\log _{9} \rho_{A}\left(x_{i}\right)-\log _{9} \rho_{B}\left(x_{i}\right)\right|$, $\left|\log _{9} \sigma_{A}\left(x_{i}\right)-\log _{9} \sigma_{C}\left(x_{i}\right)\right| \geq\left|\log _{9} \sigma_{A}\left(x_{i}\right)-\log _{9} \sigma_{B}\left(x_{i}\right)\right|,\left|\log _{9} \tau_{A}\left(x_{i}\right)-\log _{9} \tau_{C}\left(x_{i}\right)\right| \geq\left|\log _{9} \tau_{A}\left(x_{i}\right)-\log _{9} \tau_{B}\left(x_{i}\right)\right|$. It is easy to get

$\sum_{i=1}^{n}\left(\frac{1}{2^{\lambda+2}}\left(\left|\log _{9} \rho_{A}\left(x_{i}\right)-\log _{9} \rho_{C}\left(x_{i}\right)\right|^{\lambda}+\left|\log _{9} \sigma_{A}\left(x_{i}\right)-\log _{9} \sigma_{C}\left(x_{i}\right)\right|^{\lambda}+\left|\log _{9} \tau_{A}\left(x_{i}\right)-\log _{9} \tau_{C}\left(x_{i}\right)\right|^{\lambda}\right) \geq\right.$

$\sum_{i=1}^{n}\left(\frac{1}{2^{\lambda+2}}\left(\left|\log _{9} \rho_{A}\left(x_{i}\right)-\log _{9} \rho_{B}\left(x_{i}\right)\right|^{\lambda}+\left|\log _{9} \sigma_{A}\left(x_{i}\right)-\log _{9} \sigma_{B}\left(x_{i}\right)\right|^{\lambda}+\left|\log _{9} \tau_{A}\left(x_{i}\right)-\log _{9} \tau_{B}\left(x_{i}\right)\right|^{\lambda}\right)\right.$

and

$\frac{1}{2^{\lambda+1}} \max \left\{\left|\log _{9} \rho_{A}\left(x_{i}\right)-\log _{9} \rho_{C}\left(x_{i}\right)\right|^{\lambda},\left|\log _{9} \sigma_{A}\left(x_{i}\right)-\log _{9} \sigma_{C}\left(x_{i}\right)\right|^{\lambda},\left|\log _{9} \tau_{A}\left(x_{i}\right)-\log _{9} \tau_{C}\left(x_{i}\right)\right|^{\lambda}\right\} \geq$

$\frac{1}{2^{\lambda+1}} \max \left\{\left|\log _{9} \rho_{A}\left(x_{i}\right)-\log _{9} \rho_{B}\left(x_{i}\right)\right|^{\lambda},\left|\log _{9} \sigma_{A}\left(x_{i}\right)-\log _{9} \sigma_{B}\left(x_{i}\right)\right|^{\lambda},\left|\log _{9} \tau_{A}\left(x_{i}\right)-\log _{9} \tau_{B}\left(x_{i}\right)\right|^{\lambda}\right\}$

Thus, we have $d_{g h d}(A, C) \geq d_{g h d}(A, B)$. Similarly, we can prove that $d_{g h d}(A, C) \geq d_{g h d}(B, C)$ holds. This completes the proof of Property 2. 